\title{
ISSN 2689-0852
}

\section{E BIOTE्⿹勹冫NOIOGY KIOSK}

JULY 2020

AROMA 


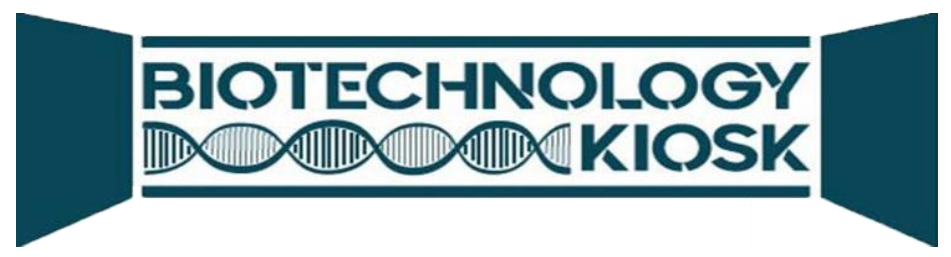

\section{Executive Publishers}

Megha Agrawal, PhD

(Biotechnology)

Publisher and Editor

Expertise:

Neuroscience, Stroke, Pharmacology, Toxicology, Microbiology and Molecular Biology

Email: megha@biotechkiosk.com meghaagra@gmail.com
Shyamasri Biswas, PhD

(Biotechnology)

Publisher and Editor

Expertise:

Structural Biology, Enzyme Technology, and Protein Crystallography

Email: shyabiswas@biotechkiosk.com shyabiswas@gmail.com

\section{Editorial, Sales \& Circulation Office}

160 International Parkway

Suite 100-9, Heathrow

FL-32746, USA

Phone: 386-518-9411

Email: publisher@biotechkiosk.com

www.biotechkiosk.com

ISSN 2689-0852

One stop shop for all things biotech

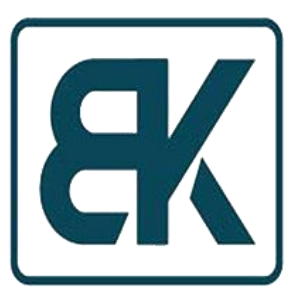




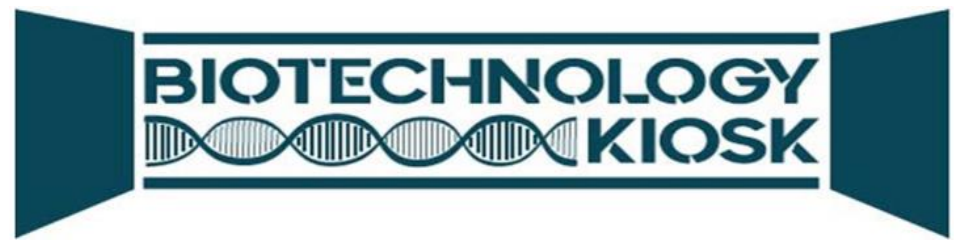

\section{From the Publisher's Desk}

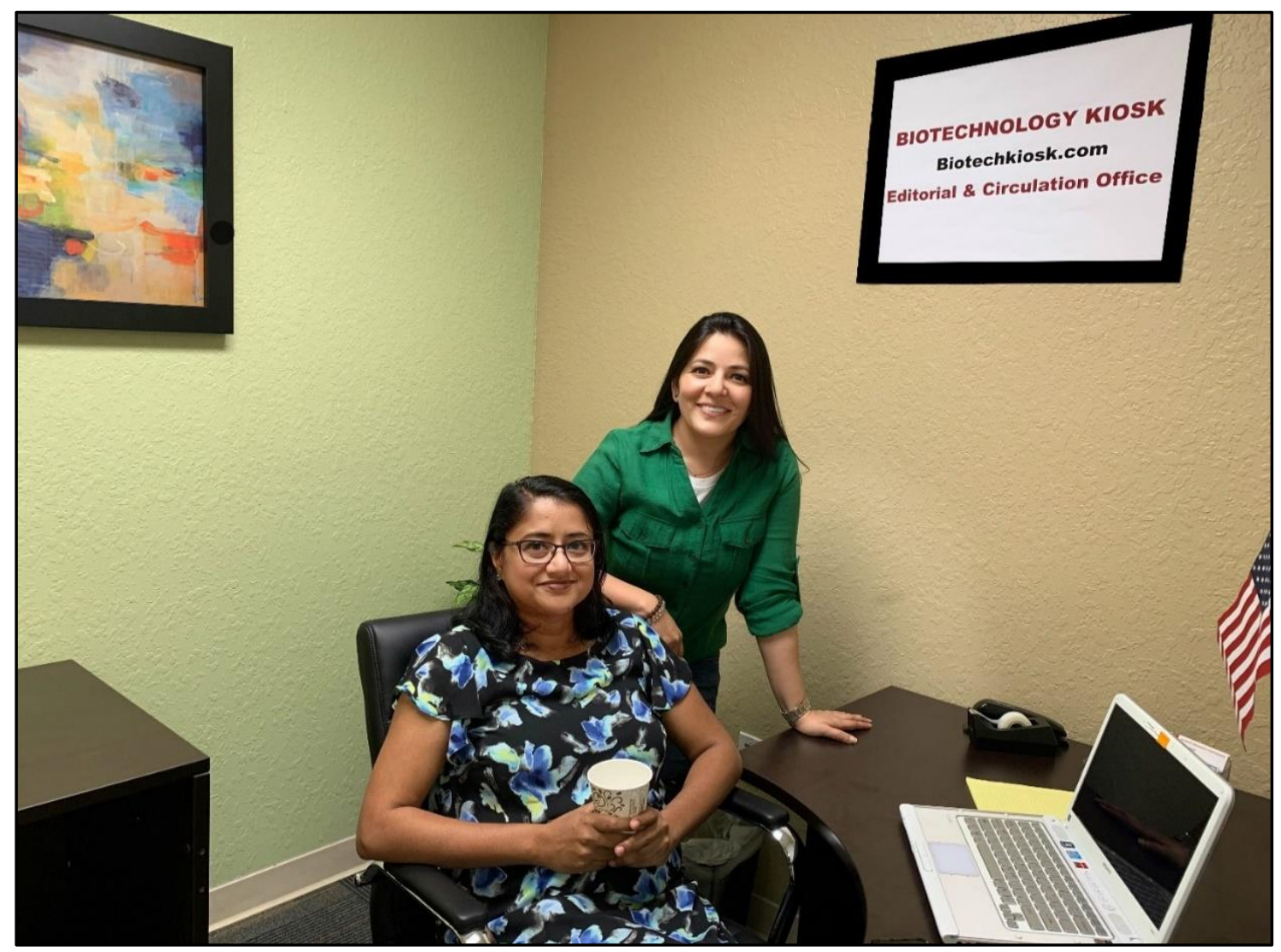

\section{Welcome to Biotechnology Kiosk!}

The July, 2020 issue of BK is now online for our readers with the regular features that include high-end editorials by experts, biotechnology advances around the world and industry news from pharma and biotech sectors.

This issue contains articles in the field of COVID-19 along with news and views on the current cutting edge topics that include latest research breakthroughs in Alzheimer's', regenerative therapies, antiaging drugs and tuberculosis and several other topics that have been discussed in the editor's choice that reports on research breakthroughs from around the world.

We look forward to receiving your feedback. We do hope that you will enjoy reading this issue of Biotechnology Kiosk. Please do write to us with your comments. Your suggestions are always appreciated.

Dr. Megha Agrawal and Dr. Shyamasri Biswas

Co Editors-in-Chief, Biotechnology Kiosk 


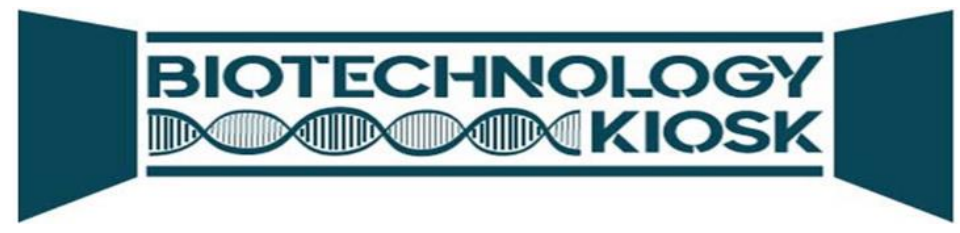

\section{Contents}

Volume 2, Issue 7

July 2020

COLUMNS

SENSORY SCIENCE

Decoding the sense of smell: understanding the structural organization within the brain https://doi.org/10.37756/bk.20.2.7.1

SEQUENCING

Next Generation Sequencing Technology in Clinical Diagnostics

https://doi.org/10.37756/bk.20.2.7.2

SENOLYTICS AND HEALTHY LIFESPANS.

Senolytics for Therapeutic Interventions for Reverse Aging and Extended Healthy Lifespans

https://doi.org/10.37756/bk.20.2.7.3

BIOTECH R\&D AND INNOVATION NEWS.

Editor's PICKS: Biotechnology Advances around the World

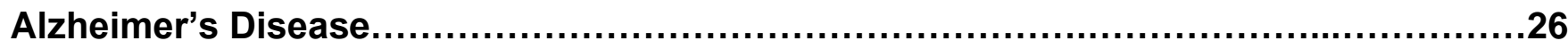

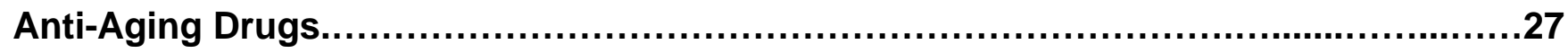

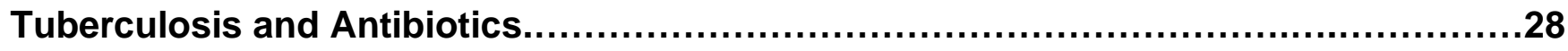

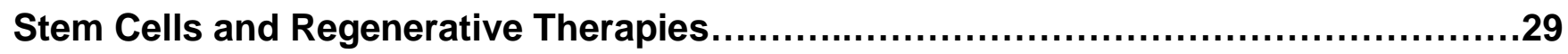

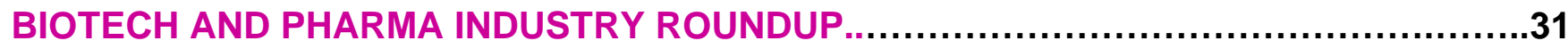

CytoSorbents got awarded \$4.4 Million for Universal Plasma Product......................31

Sangamo, Novartis join hands to develop genetic medicines for autism..................31 
Supply of Gilead's COVID-19 drug remdesivir secured by Europe..........................31

Eastman Kodak to help manufacturing drugs, vaccines in the US.........................31

AlloVir Raises \$276 Million to Advance Cell Therapy Programs............................32

GSK and Sanofi deal with U.S. Government for COVID-19 Vaccine.........................32

COVID-19 Vaccine Candidate into Phase I by Merck in Q3....................................32

Roche's IL-6 receptor blocker fails in Phase III trial...........................................

COVID-19 vaccine by Pfizer and BioNTech's shows positive data ...........................33

A New H1N1 Strain can trigger another global pandemic..................................33

ZILXI $^{\mathrm{TM}}$ (minocycline), receives FDA approval for rosacea.................................33

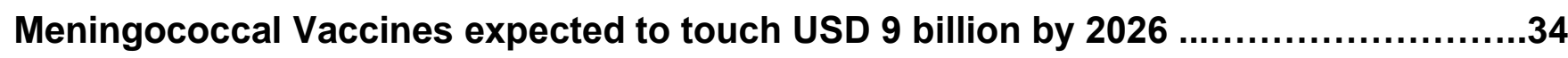

Price set for COVID-19 drug remdesivir.........................................................34

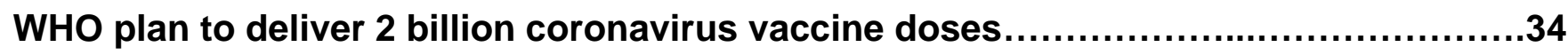

Large scale phase $2 \& 3$ trials are underway for coronavirus vaccine.......................34

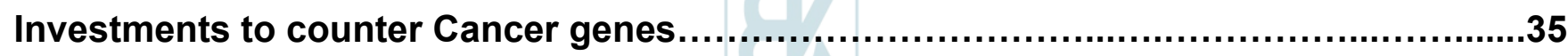

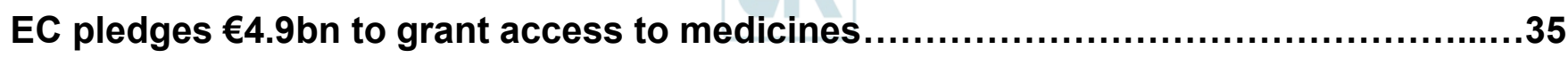

No beneficial effect of lopinavir-ritonavir on COVID-19 patients ................................

HALIX B.V. to produce vaccines against COVID-19..........................................

SARS-CoV2 antibody tests might not be suitable for individual diagnosis..................35

Enthera Pharmaceuticals Srl secures $€ 28 \mathrm{~m}$ in Series A financing............................35 


\title{
COLUMN
}

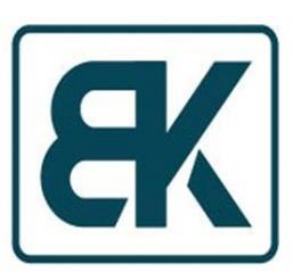

Sensory Science

By Shripriya Singh, PhD

Senior Contributing Editor

Biotechnology Kiosk, 2, 7 (2020)

DOI: https://doi.org/10.37756/bk.20.2.7.1

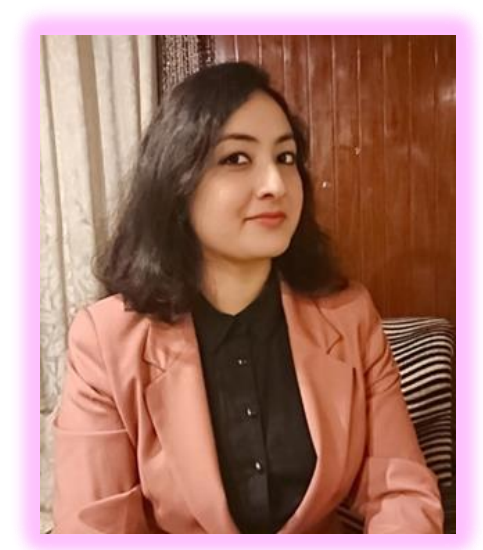

\section{Decoding the sense of smell: understanding the structural organization within the brain}

\begin{abstract}
The olfactory sense is a potent sensory tool which helps us perceive our environment much better. However, smells despite being similar have different impacts on individuals. What makes one odor categorically different from the other and why do people have a unique and personalized experience with smell is an answer that needs to be addressed. In the present article we have discussed the research in which neuroscientists have decoded and described how the relationships between different odors are encoded in the brain. How the brain transforms information about odor chemistry into the perception of smell is a major highlight of this publication. Carefully selected odors with defined molecular structures were delivered in mice and the neural activity was analyzed. It was observed that neuronal representations of smell in the cortex reflected chemical similarities between odors, thus allowing the brain to categorize scents. The study has employed chemo informatics and multiphoton imaging in the mouse to demonstrate both the piriform cortex and its sensory inputs from the olfactory bulb represent chemical odor relationships through correlated patterns of activity. The research has given us cues in the direction of how the brain translates odor chemistry into neurochemistry and eventually perception of smell.
\end{abstract}

Keywords: smell; odor; olfactory bulb; piriform cortex; brain

\section{*E-Mail: shripriyasingh@gmail.com}




\section{Introduction}

Amongst all five senses the olfactory sense is by far the most underestimated; however, we cannot ignore this potent sensory tool which helps us perceive our environment much better. Hellen Keller rightly said "Smell is a potent wizard that transports you across thousands of miles and all the years you have lived."

We as living organisms encounter various odors on a day to day basis; some odors make us tweak our noses with disgust while others make us want to whiff more. Just imagine how appealing is the smell of your favorite dish when you are hungry or how refreshing is the scent of roses when you walk through a garden. Remember the last time you complimented your special someone on how nice they smell or the time when you used aroma candles to sooth your senses. Well no matter how ignorant we are towards odors some of them will always capture our attention. Almost everyone has a preference for fragrances and what appeals to one may not smell good for the other. We often hear people talking about their signature perfumes and favorite scents, so does it simply mean people have different olfactory senses or is the perception of odors different. Well we all know that garlic will smell like garlic but it will be very different smelling from an orange. There is never a debate on this but then what makes us super sensitive towards certain odors. Fragrances and odors have the capability of evoking nostalgia, memories, romance, associations and much more. So, did you ever ponder over this simple fact that why is odor such a powerful sensory stimulant? Well the answer lies in the brain...literally!
In a novel research, neuroscientists have decoded and described how the relationships between different odors are encoded in the brain. The study helps us understand the underlying mechanism which enables individuals to have a common yet highly personalized experience with smell. How the brain transforms information about odor chemistry into the perception of smell is a major highlight of this publication. A team of neurobiologists from Harvard Medical School have provided us with new insights and helped us solve the mystery of scent. We shall briefly discuss the research recently published in Nature which describes for the very first time how different odors and their relationships are encoded in the region of brain responsible for processing smell, the olfactory cortex (1).

\section{Understanding the research}

Carefully selected odors with defined molecular structures were delivered in mice and the neural activity was analyzed. The team observed that neuronal representations of smell in the cortex reflected chemical similarities between odors, thus allowing the brain to categorize scents. Moreover, these representations can be rewired by sensory experiences. Animals are able to perceive the chemical nature of the environment via smell. The odor molecules are detected by the sensory neurons in the nose which relays the signal to the olfactory bulb (2). The olfactory bulb is a structure in the forebrain responsible for the initial odor processing (3). From here the information is transmitted to the piriform cortex, the main structure of the olfactory cortex, for further comprehensive processing (4). The present study has employed the use of chemo informatics and 
multiphoton imaging in the mouse to demonstrate both the piriform cortex and its sensory inputs from the olfactory bulb represent chemical odor relationships through correlated patterns of activity.

Sound and light stimuli can be controlled by changing characteristics such as wavelength and frequency but it is difficult to understand how the brain translates the stimulus of odor to build a neural representation of the small molecules. Even small and subtle chemical changes such as the number of carbon or oxygen atoms can alter the molecular structure of the odor molecules and thus alter the perception of smell. The team of researchers led by Sandeep Robert Datta approached this challenge focusing on the question of how the brain identifies related but distinct odors. "The fact that we all think a lemon and lime smell similar means that their chemical makeup must somehow evoke similar or related neural representations in our brains," Datta said.

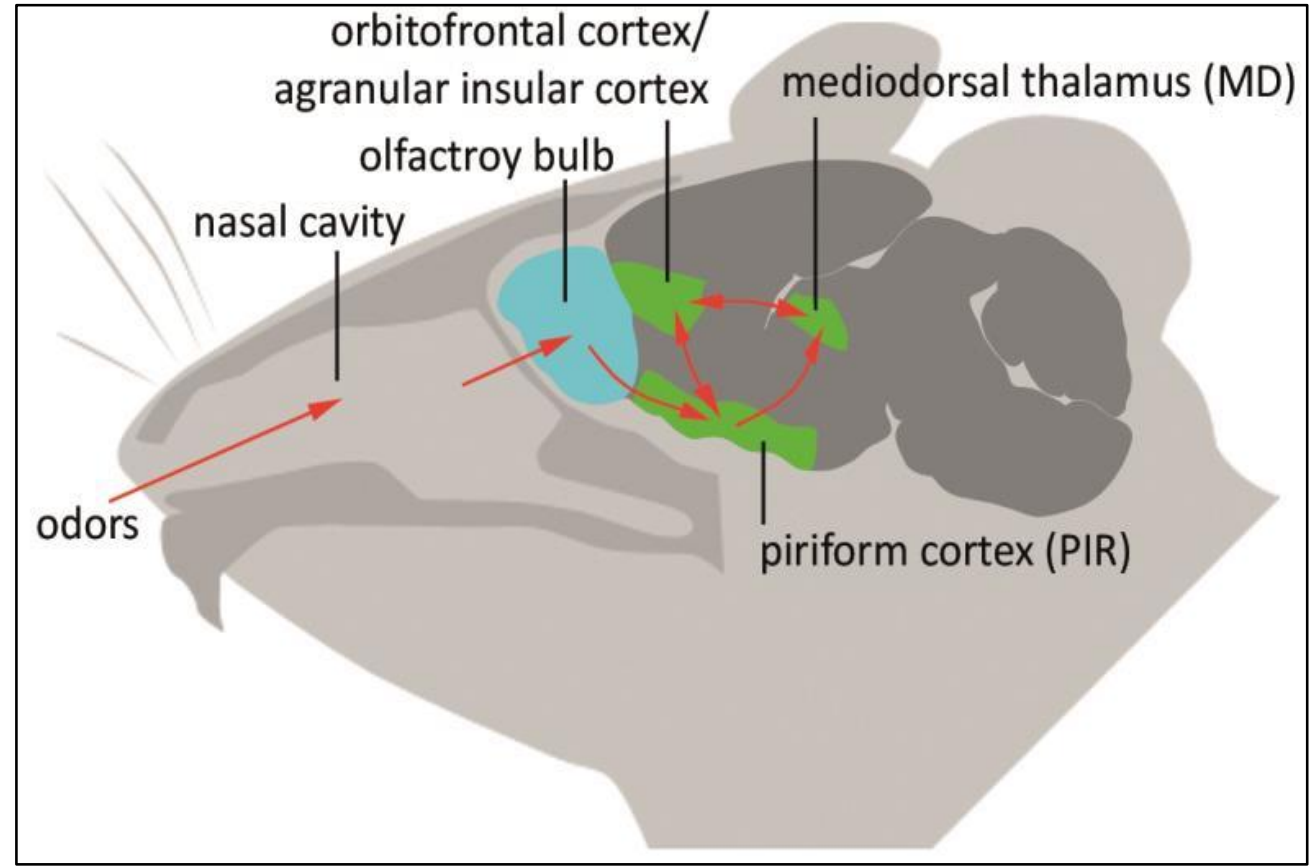

Figure 1: Diagrammatic representation of transduction of olfactory stimuli in mice. [Source: $A$ Paleocortico-Thalamo-Cortical Circuit Operating Giant Synapses; Thesis by Patric Pelzer. 2016]

First of all, the researchers tried to quantify the odor chemicals based on properties like molecular weight, the number of atoms and electrochemical properties and then using chemo informatics it was systematically computed how similar or different these odors are. The chemically defined odors were categorized into three sets namely a set with high diversity; one with intermediate diversity, with odors divided into related clusters; and one of low diversity, where structures varied only by incremental increases in carbon-chain length. Coming to the biological part, various combinations of 
odors from the different sets were then tested on mice and after being exposed to these odors multiphoton microscopic imaging was used to detect patterns of neural activity in the piriform cortex and olfactory bulb (1). Figure 1 is a schematic of transduction of olfactory stimuli in mice.

The results clearly showed similarities in odor chemistry were mirrored by similarities in neural activity. Odors that were related produced a correlated neuronal pattern both the olfactory bulb and the piriform cortex and this could be measured by overlaps in neuron activity. In contrast weakly related activity patterns were evoked by weakly related odors. The results also showed that strongly clustered patterns of neural activity were evoked in the cortex in relation to related odors as compared with the olfactory bulb. This observation remained same throughout experiments on different sets of mice. Cortical representations of odor relationships were so well-correlated that they could be used to predict the identity of a heldout odor in one mouse based on measurements made in a different mouse. Upon analysis it was discovered that patterns of neural activity were linked to a diverse array of chemical features such as electrochemical properties and molecular weight. The information gathered from the experiments is robust, reproducible and potent enough to predict cortical responses to an odor across various species. It was further observed that these neural representations were flexible. Mice were then repeatedly given a mixture of two or different odors, and over time, the corresponding neural patterns of these odors in the cortex became more strongly correlated. This process repeated even when the two odors had dissimilar chemical structures. It is the networks of neurons that selectively reshape odor relationships and thus allow the cortex to adapt to different odors. When the normal activity of these networks was blocked, the cortex encoded smells more like the olfactory bulb. "We presented two odors as if they're from the same source and observed that the brain can rearrange itself to reflect passive olfactory experiences," according to Datta. According to him lemons and oranges smell similar partially because same species have similar genomes and therefore have similarities in perception of smell. However, different individuals will have personalized perceptions as well owing to the unique genetic makeup of every human being.

Further quoting Datta, "The plasticity of the cortex may help explain why smell is on one hand invariant between individuals, and yet customizable depending on our unique experiences."

The visual and auditory cortices are relatively well understood in comparison to how the olfactory cortex converts information about odor chemistry into the perception of smell. Identifying how the olfactory cortex maps similar odors now provides new insights that inform efforts to understand and potentially control the sense of smell, according to the authors. 
Tbx21-Cre;

PHP.eB hSynapsin1-FLEx-axon-GCaMP6s
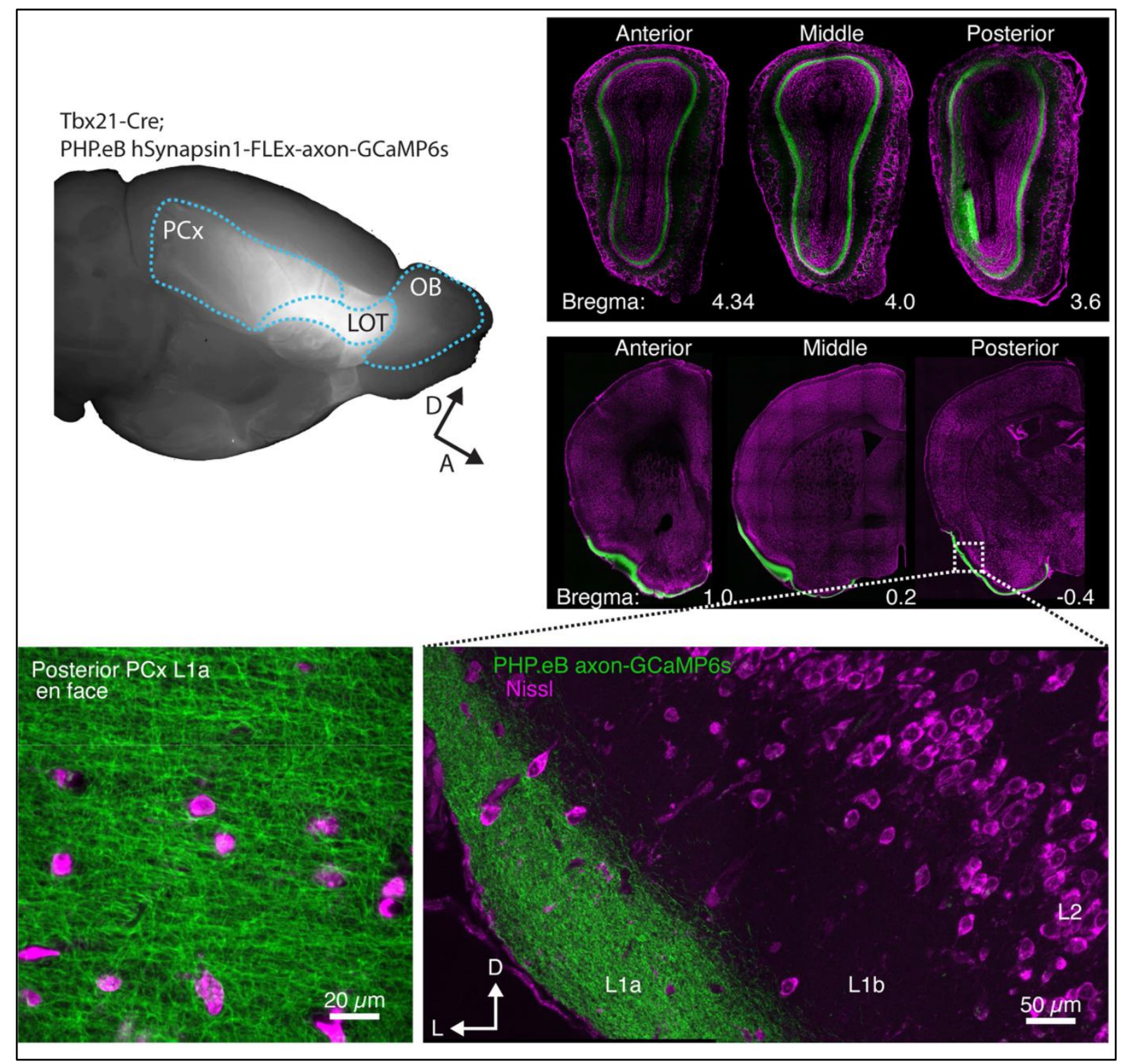

Figure 2: Functional imaging of Olfactory Bulb axons in Piriform Cortex via axonally targeted GCaMP6s. Multiphoton microscopy used in mice expressing the fluorescent $\mathrm{Ca2}+$ indicator GCaMP6s to assess neural activity in response to various odor chemicals. [Source: Nature 2020.]

\section{Concluding Remarks}

The research has clearly given us cues in the direction of how the brain translates a chemical odor molecule as an olfactory stimulus and how the neuronal networks interact to give us a comprehensive perception of smell. Any research can be best explained in the words of the original researcher and thereby we quote the authors who said "We don't fully understand how chemistries translate to perception yet." "There's no computer algorithm or machine 
that will take a chemical structure and tell us what that chemical will smell like." "To actually build that machine and to be able to someday create a controllable, virtual olfactory world for a person, we need to understand how the brain encodes information about smells," "We hope our findings are a step down that path." Thus, we hope that in our near future we shall be able to clearly understand how odor chemistry translates into neurochemistry and helps us perceive our environment much better. With this we have made a small attempt to shed a neuroscientific view on the ever-fascinating mystery of scent and odors. Till then we shall continue to dig our noses deep inside neuroscience till we can smell out potent and brain stimulating answers.

\section{References}

1. Pashkovski SL, et al. (2020) Structure and flexibility in cortical representations of odour space. Nature:1-6. DOI: https://doi.org/10.1038/s41586-0202451-1

2. Sosulski DL, Bloom ML, Cutforth $T$, Axel $R$, \& Datta SR (2011) Distinct representations of olfactory information in different cortical centres. Nature 472(7342):213-216. DOI: https://doi.org/10.1038/nature09868

3. Miyamichi K, et al. (2011) Cortical representations of olfactory input by trans-synaptic tracing. Nature 472(7342):191-196. DOI: https://doi.org/10.1038/nature09714

4. Haberly LB (2001) Parallel-distributed processing in olfactory cortex: new insights from morphological and physiological analysis of neuronal circuitry. Chemical senses 26(5):551-576. DOI:

https://doi.org/10.1093/chemse/26.5.551 


\title{
COLUMN
}

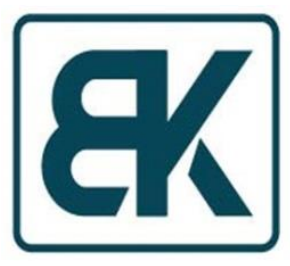

Sequencing

By Megha Agrawal*, PhD

Co Editor-in-Chief

Biotechnology Kiosk, 2, 7 (2020)

DOI: https://doi.org/10.37756/bk.20.2.7.2

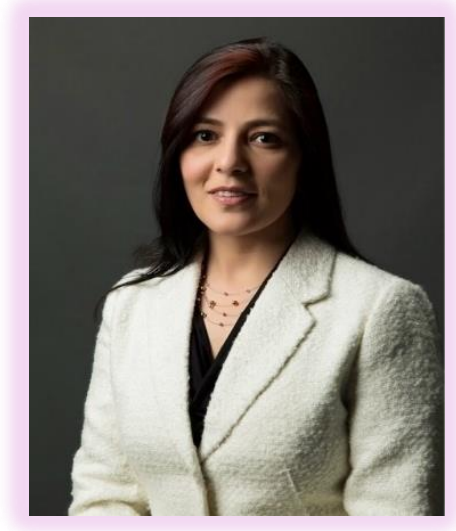

\section{Next Generation Sequencing Technology in Clinical Diagnostics}

\begin{abstract}
The promise of next generation sequencing offers hope for the current DNA sequencing technology to evolve from the applications in basic research to transition to the clinical diagnostics. This advancement in the sequencing technology is happening in part due to the introduction of high throughput and benchtop instruments that offer fully automated cost-effective sequencing along with faster assay times. This development is believed to remove the bottleneck of the complex and cumbersome library preparation that include isolation of nucleic acids and the resulting amplified and barcoded DNA with sequencing adapters. Here, we present a brief overview of the principles of next generation sequencing and automation of library preparation along with the diagnostic applications of next generation sequencing in human immunogenetics. Finally, an outlook is presented.

Keywords: microfluidics, multiplexing, genome, NGS.
\end{abstract}

*E-Mail: megha@biotechkiosk.com

To cite this article: Agrawal M; Next Generation Sequencing Technology in Clinical Diagnostics, Biotechnology Kiosk, Vol 2, Issue 7, PP: 10-17 (2020); DOI: https://doi.org/10.37756/bk.20.2.7.2 
Introduction: The Process of Next Generation Sequencing

Over the last few decades, there has been tremendous growth in DNA sequencing technology. This growth has witnessed the evolution of the technology in phases that include the discovery of the double helix DNA shape and the complete sequencing of a human genome along with a continuously widening range of applications in research, agriculture and public health [1-4]. Currently, next generation sequencing (NGS) is employed to sequence complete genomes. However, the usual costs of sequencing a human genome are somewhat high that lie around the \$1000-mark [4]. Researchers have shown that to reduce the costs, a viable way is to increase the throughput of machines. This has resulted in a decrease in sequencing costs per base [4]. A number of studies have shown major successes with regard to reliability along with data that are considered prerequisites for NGS to be applied to clinical diagnostics $[4,5]$.

Figure 1 schematically shows the various process elements involving the NGS. These include sample pre-processing, library preparation, sequencing itself and bioinformatics [4]. It is to be noted that all modern sequencing technologies require dedicated sample preparation to yield the sequencing library loaded onto the instrument regardless of the underlying principles of the respective sequencing method [4]. The NGS process involves sequencing libraries that consist of DNA fragments of a defined length distribution with oligomer adapters at the $5^{\prime}$ and $3^{\prime}$ end for barcoding along with the actual sequencing process. Subsequently, the generated data is analyzed using bioinformatics after sequencing (Figure 1) [4].

Routine laboratory practice in NSG is very important that involves reliable and standardized implementation and also quality control measures for all stages of the NSG process [4]. There are some challenges that are encountered at each of the workflow steps shown in Figure 1. Especially, during library preparation, major challenges include complexity of protocols, contamination and cost [4]. Researchers have shown that the extraction of a sufficient amount of DNA from the sample input without extracting disturbing inhibitors can result in changing the complexity depending on the sample material. Further, carry-over contamination can occur during sequencing runs that can lead to significant errors [6]. In addition, a significant challenge involves the bioinformatics component in the NGS. Studies have shown that the handling of the extremely large amounts of data generated by high-throughput NGS often requires considerable information technology (IT) resources. However, for such comprehensive analysis, there are now enough advanced IT solutions available [4]. These challenges must be overcome to ensure high quality sequencing results [4].

Studies have shown an inherent problem with sample contamination as libraries are usually prepared in parallel [6]. It has been shown that the costs incurred during library preparation derive from laboratory equipment, trained personnel's hands-on time and reagent cost [4]. 


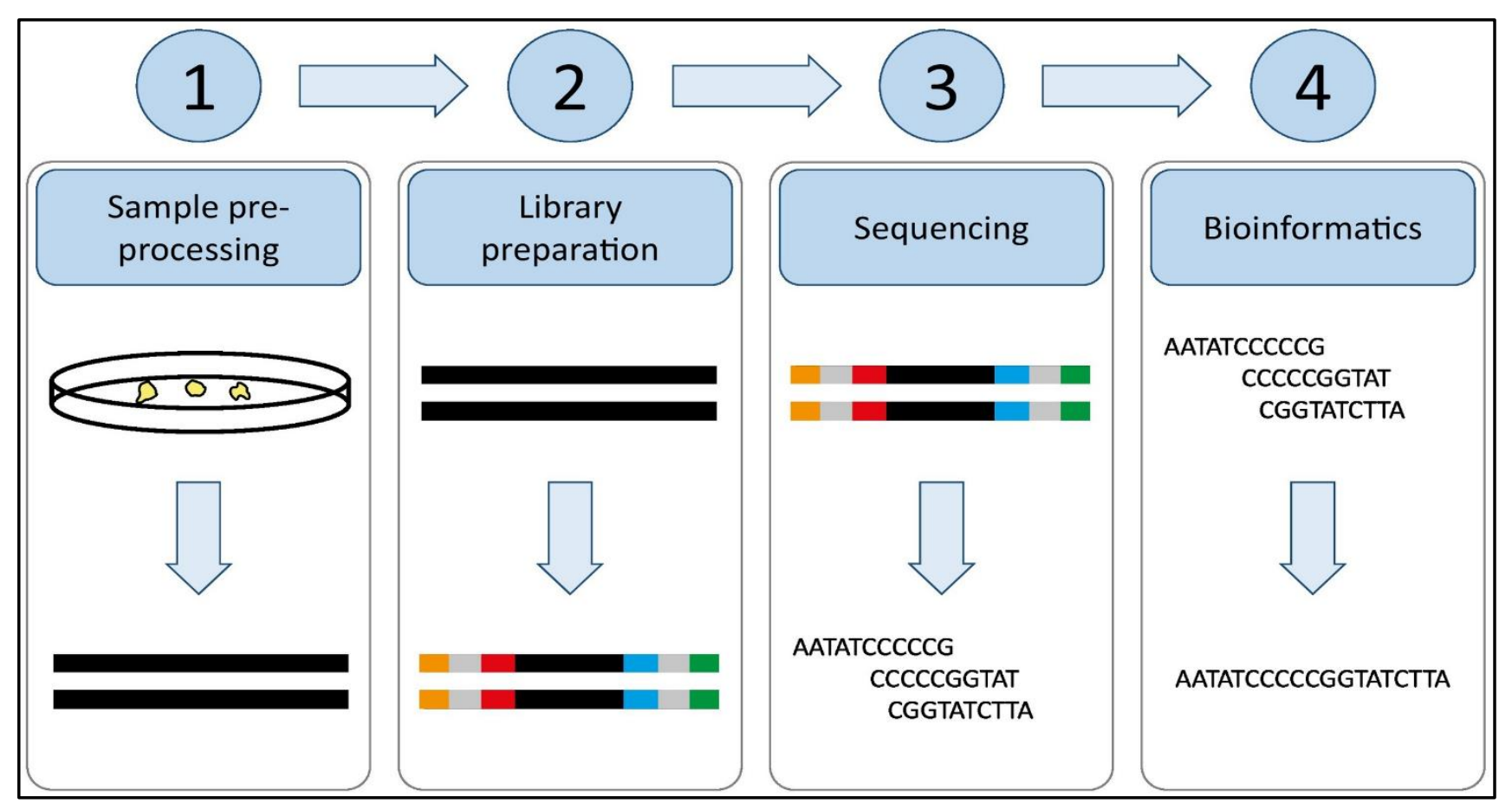

Figure 1: Schematic depiction of workflow for NGS that shows extraction of the nucleic acid from the sample (e.g. blood, sputum, bone marrow, bacterial cultures, etc.). This is followed by library preparation that yields a population of DNA fragments of defined lengths with defined oligomer sequences at both ends to be compatible with the applied sequencing technique. Subsequently, the actual sequencing run occurs on the respective system. Resulting sequence data is then processed by a bioinformatics analysis pipeline [Source: Biotechnology Advances, 2020].

The Automation: Advanced Library Preparation and Performing Complex Protocols

Previous studies have suggested that an automation based NGS process can provide the viable solution to perform complex protocols with high reproducibility, leading to reduced error rates [7]. In general, there are four main scenarios for which automation of library preparation can be considered. These include sequencing centers, diagnostics laboratories, point-of-care and research (Figure 2) [4].
The biotechnology research and industry has already employed sequencing centers and diagnostic laboratories. For future technology development, the point-ofcare components are predicted opportunities of NGS [4]. Further, it is envisioned that microfluidic solutions could be an attractive option in sequencing centers along with pipetting workstations that could be used in research (Figure 2). It has been shown that pipetting workstations can be used for high throughput scenarios. Whereas, microfluidic solutions can be employed for low throughput applications [4]. 
It has been shown by reducing human interaction with the reagents and samples contamination risk can be significantly lowered. This also leads to decreased costs per sample due to the fact that hands-on time is reduced or fewer reagents are used due to miniaturization [4]. However, there are some disadvantages of automation techniques due to the involvement of high number of resources that are needed to initially provide and implement the instruments in the laboratory workflow and the costs associated with the maintenance. Despite these issues, researchers have shown that profitable efficiency can be achieved by using the system intensively. Another route of profitability is going for low investments and also if high prices are paid for high quality analysis [4].

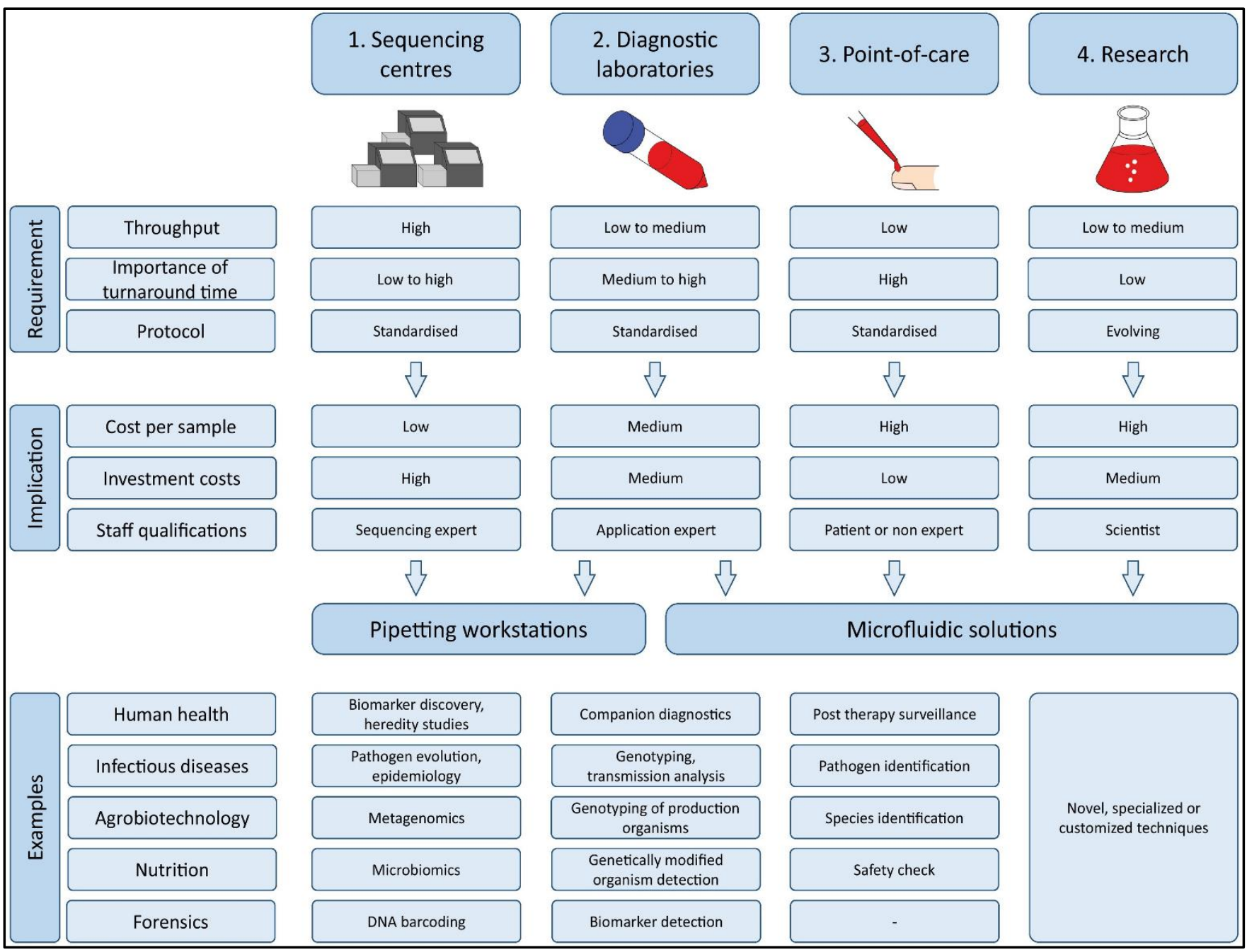

Figure 2: An illustrative overview of four different sequencing scenarios for library preparation for next generation sequencing (NGS). These scenarios differ in terms of associated requirements that include throughput, protocol and importance of turnaround time. Potential examples for different domains are also shown at the bottom of the figure [Source: Biotechnology Advances 2020]. 
Microfluidics

Based

Automation Solutions

There are multiple advantages of using microfluidics. Microfluidics based operation represents an interesting alternative for automation of library preparation due to the fact that it enables miniaturization, automation and parallelization of biochemical analysis [4]. Studies have shown that the assignment of the genetic information to the cell of origin is a technical challenge within single cell analysis by sequencing. This can be overcome by the application of microfluidics. Especially, droplet microfluidics have been shown to offer unique solutions when larger cell numbers are analyzed (>104) [4]. To this end, researchers employed encapsulated single cells in single beads that provided unique barcodes. Two aqueous flows that contained the cells and the beads respectively were merged shortly before compartmentalization. After droplet generation, cells were lysed and mRNA hybridized on primers that were immobilized on the bead surface [4]. Beads were then recovered and washed manually and subsequently reverse transcriptase transferred the mRNA into cDNA that was amplified by PCR (Figure 3) [4].

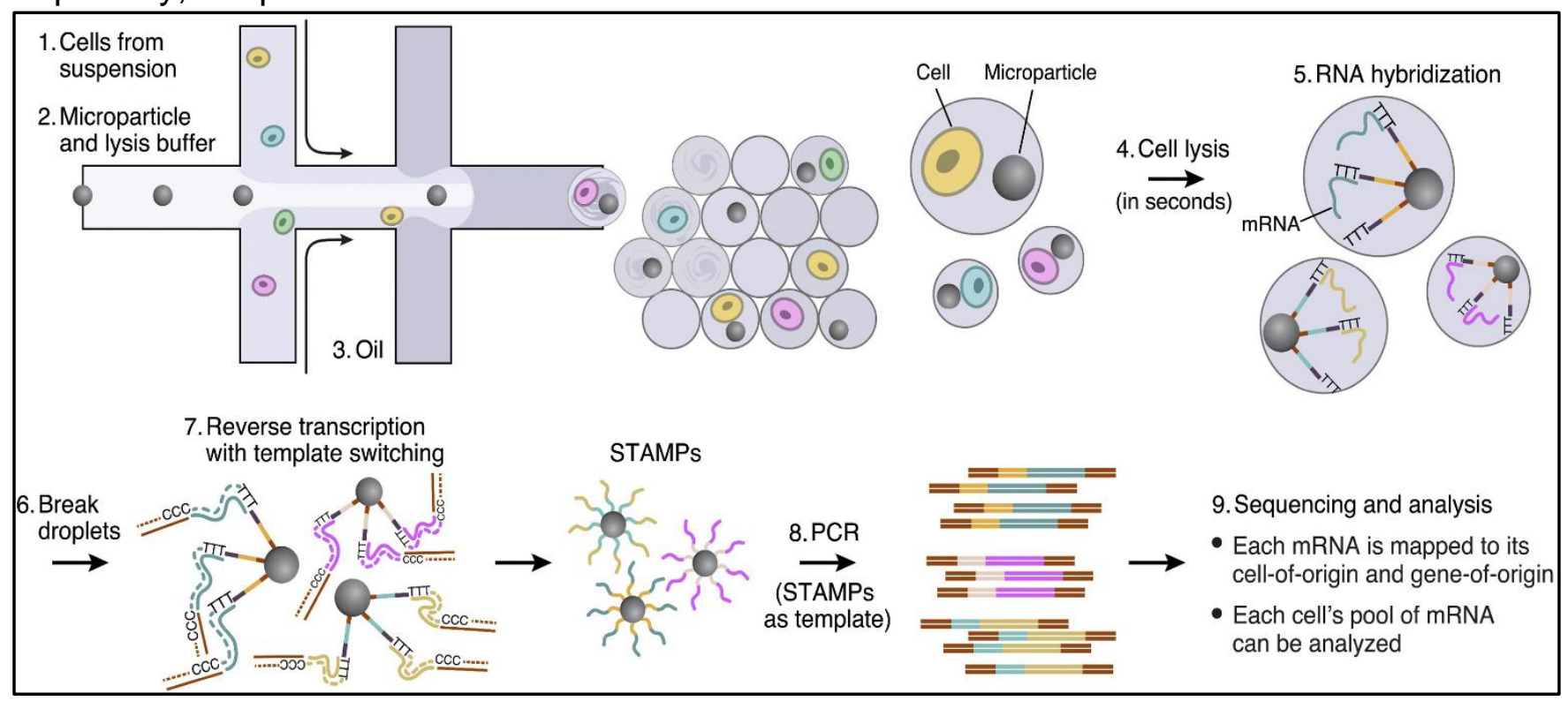

Figure 3: Illustrations showing single-cell barcoding with automated microfluidics solutions with additional features for library preparation [Source: Biotechnology Advances, 2020].

\section{Next Generation Sequencing for Human Immunogenetics}

Protein-coding sequences have been most widely studied that represent the best understood part of the human genome.
However, the non-coding sequence is known to be substantially larger that constitutes about $98 \%$ of the human genome [8]. Researchers have suggested difficulty in the interpretation of any genetic findings for noncoding DNA. Figure 4 shows the exome 
sequencing focusing on the protein coding parts of genes, which is more widely used in human genomics than whole genome sequencing $[4,8]$. This approach has been shown to require exome enrichment of the sequencing library that is essentially the capture of the DNA sequences containing the protein-coding regions [4]. Because the sequencing output is limited in this step, it reduces the sequencing cost along with the amount of effort required to analyze and interpret the data [4]. Diagnostic applications are shown in Figure 4. It uses restricted approaches that involve targeted 'panel' sequencing. In this configuration, only certain genes or smaller gene regions of interest are captured and sequenced, usually with a high coverage [4]. These advances have led to the development of NGS-based gene panels that are currently regularly used in diagnostic laboratories and a number of companies and institutions provide panel tests for different types of diseases [4].

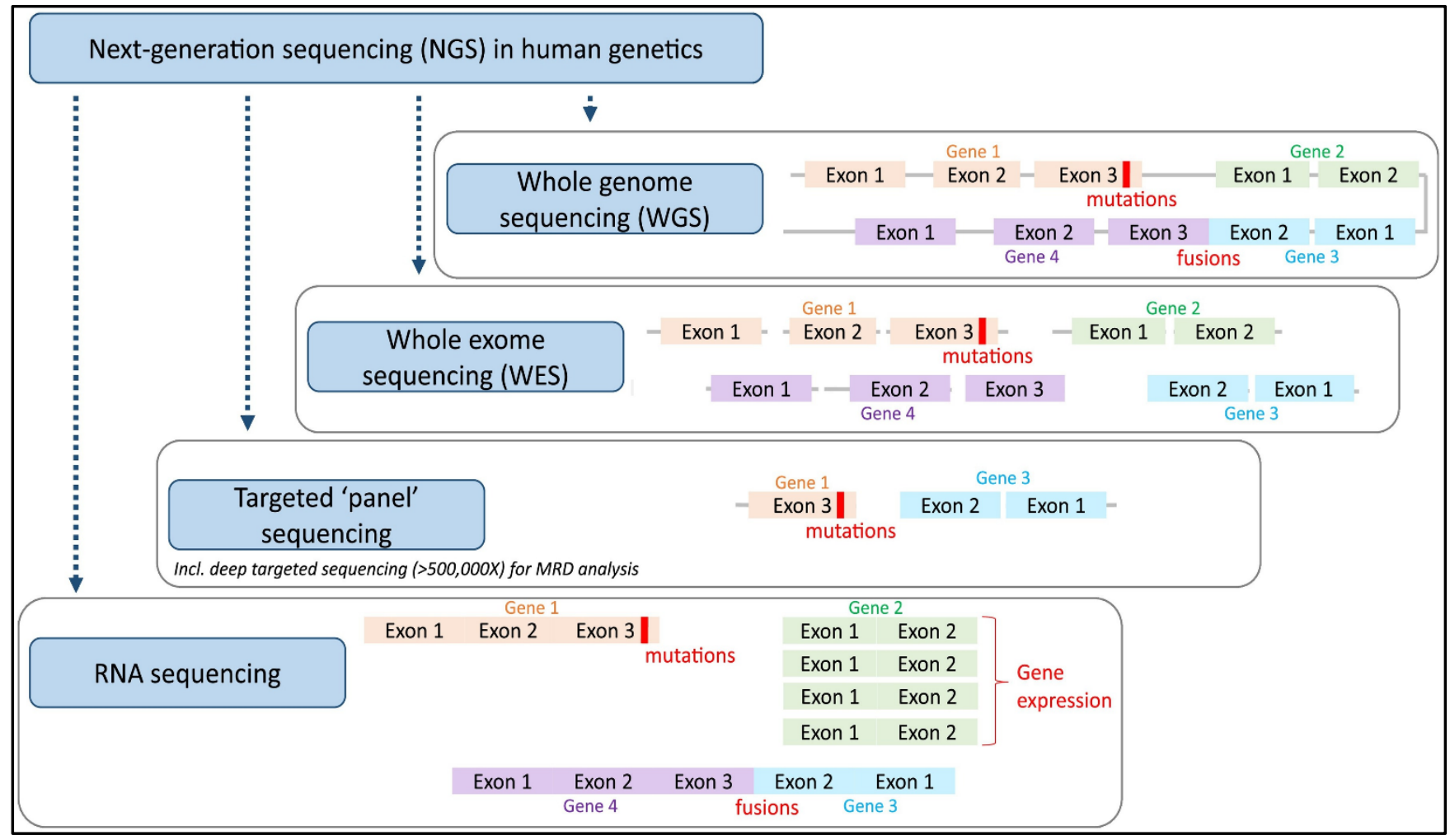

Figure 4: Next generation sequencing diagnostic applications in human genetics showing different sequencing targets for sequencing in human genetics. These involve whole genome sequencing, whole exome sequencing, targeted panel sequencing and RNA sequencing [Source: Biotechnology Advances, 2020].

\section{Conclusion and Outlook}

New generation sequencing (NGS) based assays are finding increasing applications in a vast array of diverse fields. However, there are challenges associated with the NGS technology that include complex and laborintensive library preparation. To overcome the challenges, different automation solutions 
have been suggested that can be considered to address the needs of each specific setting. To this end, the well-established pipetting workstations are usually suggested for commercially available systems for high throughput applications.

Researchers have also paid attention to microfluidic devices that are emerging as an enabling technology for single cell analysis and as an alternative for lower throughput applications. These technologies are associated with lower capital costs that simplify their entry into the automation arena. However, before these technologies make into the commercialization stages, there are still scopes for further improvement in the standardization and reproducibility factors. It is anticipated that future developments for diagnostic laboratories and point-of-care applications would employ microfluidic systems that could considerably improve performance and ease-of-use of sequencing approaches for various advanced biomedical applications.

\section{References:}

1. E.S. Lander, L.M. Linton, B. Birren, C. Nusbaum, M.C. Zody, J. Baldwin, et al., Initial sequencing and analysis of the human genome, Nature, 409 (6822), 860921 (2001),

DOI:

https://doi.org/10.1038/35057062

2. Johannes R. Lemke, Erik Riesch, Tim Scheurenbrand, Max Schubach, Christian Wilhelm, Isabelle Steiner, Jörg Hansen, Carolina Courage, Sabina Gallati, Sarah Bürki, Susi Strozzi, Targeted next generation sequencing as a diagnostic tool in epileptic disorders, 53, 8, 1387-1398 (2012), https://doi.org/10.1111/j.1528$\underline{1167.2012 .03516 . x}$

3. Sarah E. Calvo, Alison G. Compton, Steven G. Hershman, Sze Chern Lim, Daniel S. Lieber, Elena J. Tucke et. al., Molecular Diagnosis of Infantile Mitochondrial Disease with Targeted NextGeneration Sequencing. Science Translational Medicine, 4, 118, pp. 118ra10 (2012), DOI: 10.1126/scitranslmed.3003310

4. J.F. Hess, T.A. Kohl, M. Kotrová, K. Rönsch, T. Paprotka, V. Mohr, T. Hutzenlaub, M. Brüggemann, R. Zengerle, S. Niemann, N. Paust, Biotechnology Advances,41, 107537 (2020), DOI: https://doi.org/10.1016/j.biotechadv.2020. $\underline{107537}$

5. Jeffrey Gagan \& Eliezer M. Van Allen, Next-generation sequencing to guide cancer therapy, Genome Medicine, 7, 80 (2015),

DOI: https://doi.org/10.1186/s13073-015-0203$\underline{x}$

6. Michaela Kotrova, Jan Trka, Michael Kneba, Monika Bru"ggemann, Is NextGeneration Sequencing the way to go for Residual Disease Monitoring in Acute Lymphoblastic Leukemia? Molecular Diagnostics and Therapy, 21:481-492 (2017),

DOI: https://doi.org/10.1007/s40291-017-0277$\underline{9}$

7. H. Fleischer, K. Thurow, Automation Solutions for Analytical Measurements: Concepts and Applications, Wiley-VCH, 
Weinheim, Germany

DOI:10.1002/9783527805297

8. [Brian S. Gloss \& Marcel E. Dinger, Realizing the significance of noncoding functionality in clinical genomics, Experimental \& Molecular Medicine 50, 97 (2018),

(2018).

\section{https://doi.org/10.1038/s12276-018-0087-} $\underline{0}$

9. Pelegrin $M$, Naranjo-Gomez $M$, Piechaczyk M. Antiviral Monoclonal Antibodies: Can They Be More Than Simple Neutralizing Agents? Trends Microbiol. 2015;23(10):653-665. DOI: $\underline{10.1016 / j . t i m .2015 .07 .005}$ 


\title{
COLUMN
}

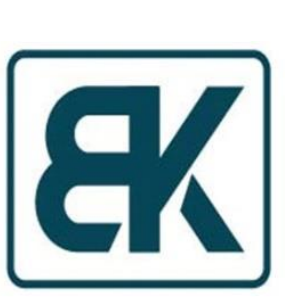

Senolytics and Healthy Lifespans

By Shyamasri Biswas*, PhD Co Editor-in-Chief

Biotechnology Kiosk, 2, 7 (2020)

DOI: https://doi.org/10.37756/bk.20.2.7.3

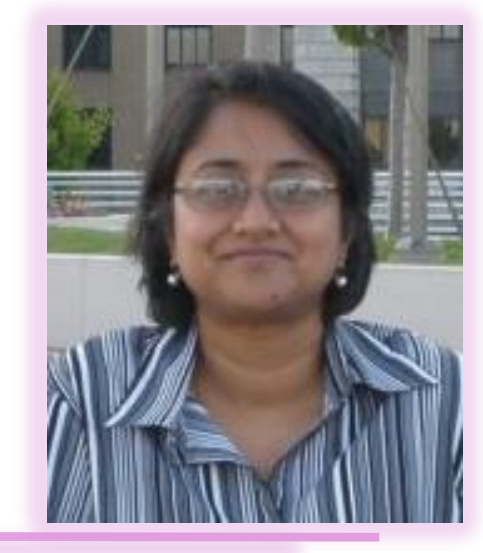

\section{Senolytics for Therapeutic Interventions for Reverse Aging and Extended Healthy Lifespans}

\begin{abstract}
Cellular senescence is believed to possess a clear hallmark of normal chronological aging. The current research focus has been to better our understanding of the fundamental causes of aging and identification of its potential therapeutic targets as well as modulators of senescence cells. This is considered of paramount importance in maintaining a lasting health and longevity. Senolytics correspond to the drug-mediated process that seek to develop modulating agents for senescence cells to delay, prevent, alleviate, or reverse age-related diseases. Here, we present some of the recent notable breakthroughs in regulating cellular senescence for a number of significant anti-aging and pro-health therapeutic applications of senolytics.
\end{abstract}

\section{*E-Mail: shyabiswas@biotechkiosk.com}




\section{Introduction: Regulating Senescent Cells}

Senescent cells are believed to accumulate with age in multiple tissues that may cause age-associated disease and functional decline. Genetic or drug-mediated specific ablation of senescent cells has been considered to ameliorate a wide range of ageassociated disabilities and diseases in mice [1-3]. The drug-mediated process is often referred to as senolytics, which seek to develop agents to delay, prevent, alleviate, or reverse age-related diseases. To this end, early research in the field demonstrated the possibility of clearing senescent cells by activating a drug-inducible 'suicide' gene that was shown to enhance health span and delay multiple age-related phenotypes in genetically modified progeroid mice [3]. It is believed that senolytics based therapeutic interventions could reduce the burden of senescent cells. This could potentially ameliorate age-related disabilities and chronic diseases as a group [4].

Thus, in view of the huge medical benefits that are associated with senolytics, current industrial research and developments indicate that a large number of pharmaceutical companies are now actively engaged in the discovery of senolytic drugs that can target senescent cells [5]. However, it is also believed that many drugs including antibiotics that are approved by the US Federal Drug and Administration (FDA) may also possess senolytic activity and by employing these drugs would dramatically accelerate the clinical use of the senolytic drugs in any anti-aging drug trials [5]. A number of research groups have studied several candidate senolytic drugs in-vitro [4].
Among most of the senolytic agents that have been tested, Dasatinib (D) and Quercetin ( $Q$ ) have been shown to be particularly promising in clearing senescent cells. Further, the combination of $\mathrm{D}+\mathrm{Q}$ has been considered very effective that can selectively target a broader range of senescent cell types. It was reported that in both senescent and nonsenescent cultured preadipocytes, $D$ and $Q$ reduced expression of the anti-apoptotic regulator PAI-2 [4].

\section{Targeting Senescent Cells with Clinically- Approved Drugs}

In a recent research, a simple but innovative senolytic screening approach was shown that allowed systematically identification of clinically-approved drugs (Figure 1) to target the senescence phenotype of human fibroblasts [5]. To achieve the goals, MCR-5 and $\mathrm{BJ}$ cells were treated with BrdU (a DNAdamaging agent). By employing this screening approach, two clinically-approved macrolide antibodies (namely, Azithromycin and Roxithromycin) were demonstrated to preferentially exhibit senolytic activity [5].

In this study, the methodology used a simplified screening assay that enabled identification and repurposing of clinicallyapproved therapeutics with senolytic activity for the treatment of aging and agingassociated disorders (Figure 1) [5]. Subsequently, two independent normal and non-immortalized human fibroblast cell lines, MCR-5 (for screening) and BJ (for validation) derived from human lung and skin tissues were employed. The responses of normal fibroblasts and senescent fibroblasts were then directly compared, and drugs that preferentially killed senescent fibroblasts 
were considered the senolytic drugs. This approach enabled identification of two erythromycin-family members, Azithromycin and Roxithromycin that preferentially targeted senescent fibroblasts (Table 1). It was observed that Azithromycin preferentially targeted and removed almost $97 \%$ senescent cells with great efficiency (Table 1) [5]. This represented a near 25-fold reduction in senescent cells. However, Erythromycin was found ineffective in any senolytic actions [5].

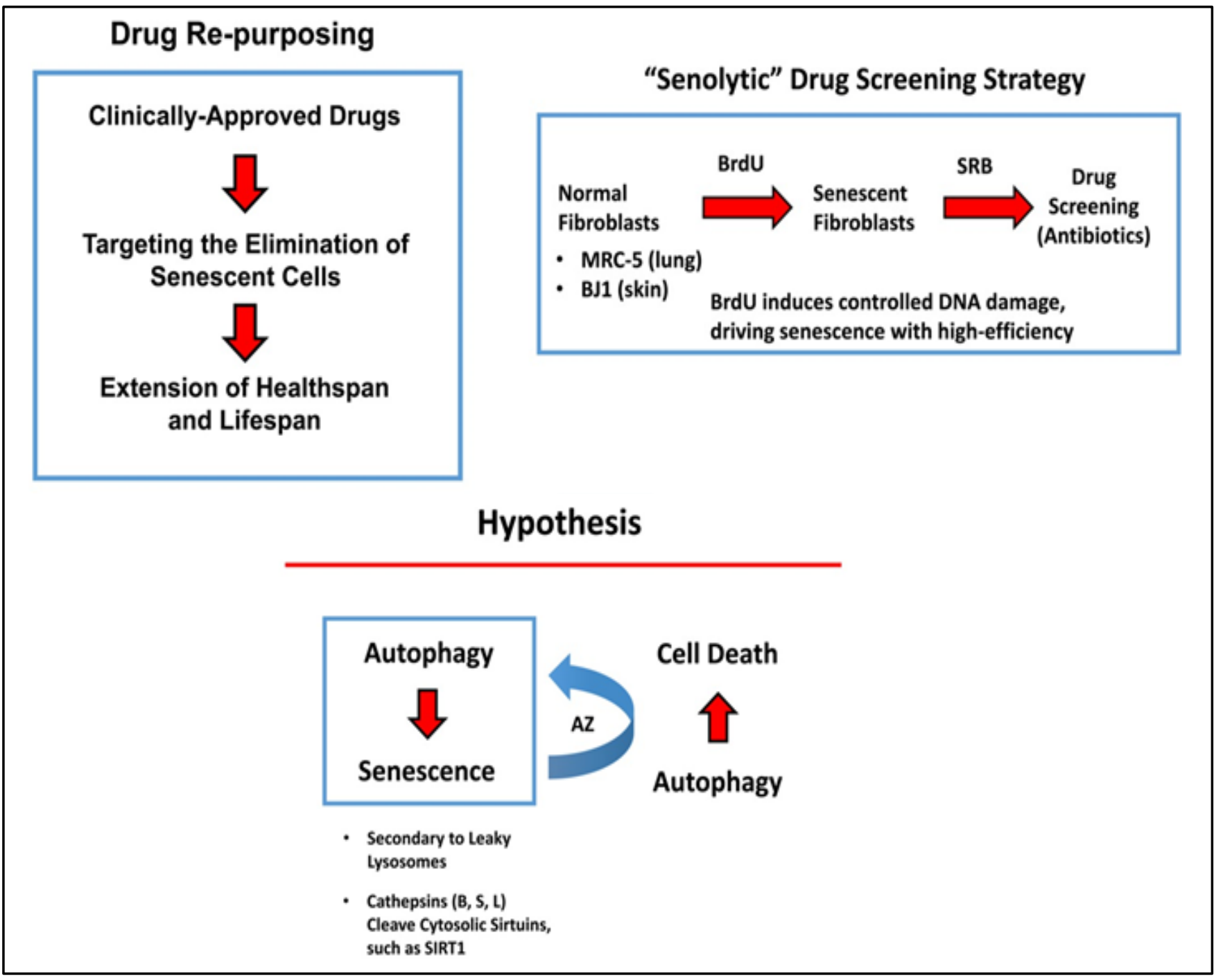

Figure 1: A schematic presentation of targeting senescent cells with clinically-approved drugs including antibiotics with the goal of increasing health span and lifespan (Left Upper Panel). The Senolytic drug screening strategy is shown to induce controlled DNA-damage and elimination of senescence (Right Upper Panel). A hypothesis is also presented on the potential role of autophagy in conferring senolytic activity of Azithromycin (AZ) (Bottom panel) [Source: AGING, 2018]. 
The hypothesis behind this study (Figure 1) was based on the previous experimental observations of activation of autophagy by inhibition of mTORC that was shown to efficiently suppress senescence phenotypes [6]. It is known that autophagic cells have greater likelihood of becoming senescent. This process is known as autophagysenescence transition (AST) [5]. It has been shown that cells accumulate auto-phagic organelles (lysosomes and autophagosomes) during autophagy. These structures are believed to involve sequestering of dangerous proteases, including the cathepsins (B, S and L) [5]. Further, once the cathepsins are in the cytosol, they can proteolytically cleave the sirtuins, such as SIRT1 that can drive the senescence pheno-type [7]. In this study, Azithromycin (AZ), which is a weak autophagy inducer is found to preferentially target senescent cells [5]. Thus, the authors hypothesized that the induction of autophagy in senescent cells can drive cell death (Figure 1) [5].

\begin{tabular}{|c|c|c|}
\hline & \multicolumn{2}{|c|}{$\begin{array}{r}\text { Viability of senescent MRC-5 cells } \\
\text { (\% vs. control) }\end{array}$} \\
\hline & $100 \mu \mathrm{M}$ & $50 \mu \mathrm{M}$ \\
\hline Erythromycin & 100 & 100 \\
\hline Azithromycin & 56.01 & 100 \\
\hline Roxithromycin & 30.99 & 100 \\
\hline
\end{tabular}

Table 1: The effects of antibiotics on BrdU-treated senescent MRC-5 fibroblasts. The biological effects of three antibiotics, namely Erythromycin, Azithromycin and Roxithromycin, on cell viability are summarized. Erythromycin was found to be completely ineffective. Roxithromycin and Azithromycin had no effect at a lower dose $(50 \mu \mathrm{M})$, but selectively eliminated large numbers of senescent cells at $100 \mu \mathrm{M}$. Azithromycin was particularly found to be the most selective compound, as it eliminated senescent cells without affecting control cells [Source: AGING, 2018]. 
Human Clinical Trial of Senolytics in Chronic Health Dysfunctions

In a more recent research on senolytics in chronic health dysfunctions, researchers reported a first-in-human clinical trial of Dasatinib (D) plus Quercetin (Q) in individuals with diabetic kidney disease [8, 9]. The combination of $D+Q$ was shown to be quite effective in selectively eliminating senescent cells. The combined senolytics power of $D+Q$ results in transiently disabling pro-survival networks that defend these cells against their own apoptotic environment [8]. This apparently first clinical trial of $D+Q$ senolytics exhibited improved physical function in patients with pulmonary fibrosis (IPF), which is a fatal senescence induced disease [8]. a

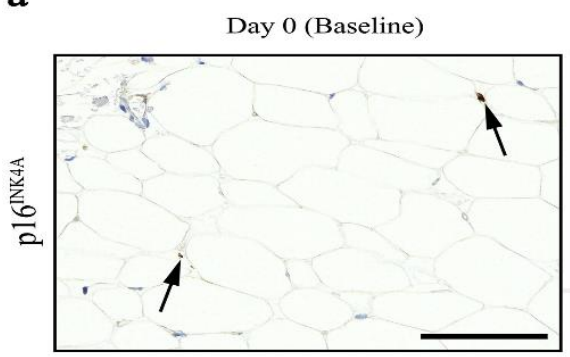

b

Day 0 (Baseline)

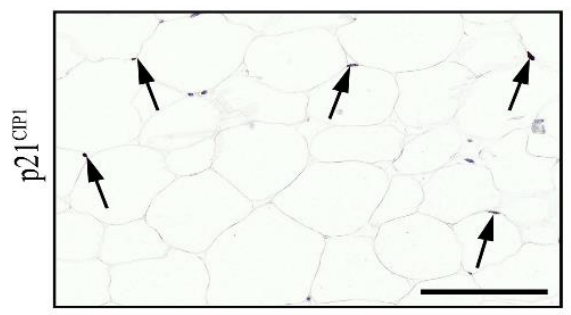

c

Day 0 (Baseline)

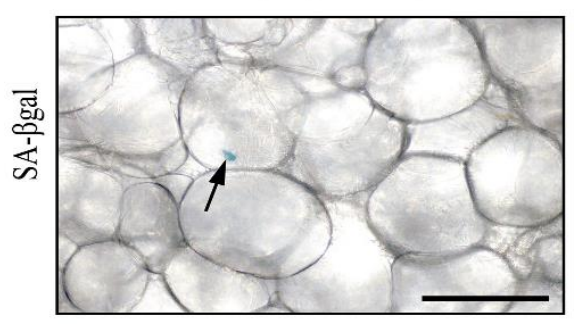

Day 14 (Post-Treatment)

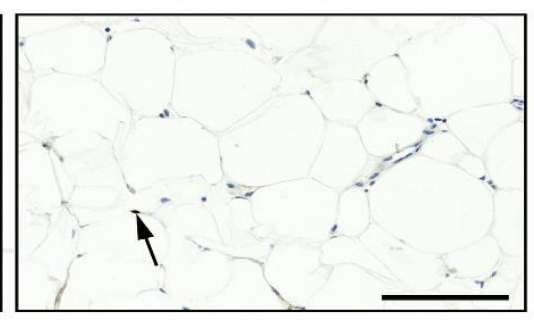

Day 14 (Post-Treatment)

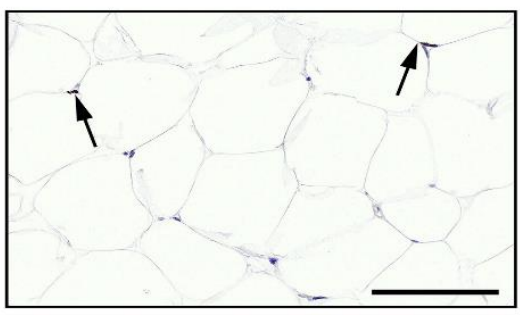

Day 14 (Post-Treatment)

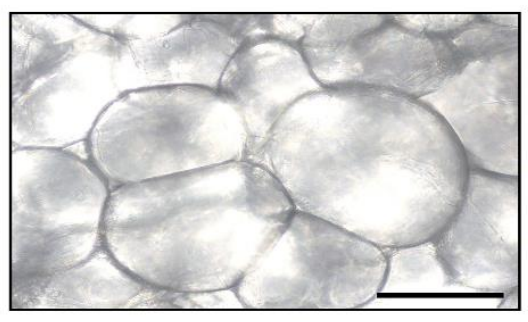

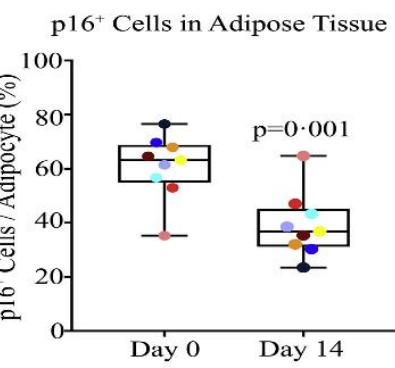

p $21^{+}$Cells in Adipose Tissue

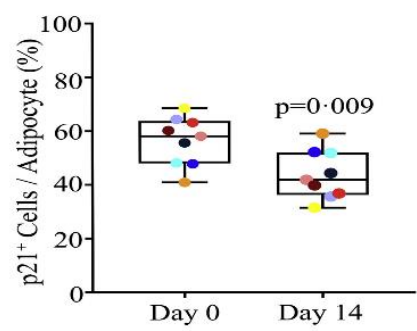

SA- ggal $^{+}$Cells in Adipose Tissue

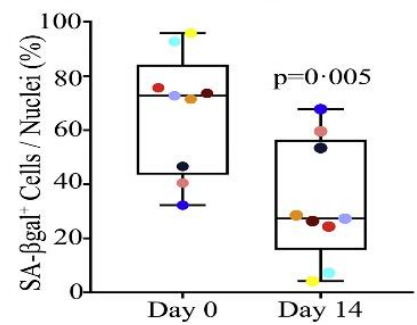

Figure 2: Clinical administration of $D+Q$ that decreases human adipose tissue senescent cells. (a) $D+Q$ significantly reduced $(p=0.001)$ abdominal subcutaneous adipose tissue $p 16^{\text {INK } 4 A+}$ cells (raw values reduced by $35 \%$ in sections of adipose tissue biopsied at Day 14). Representative images can be seen. (b) $D+Q$ significantly reduced $(p=0.009)$ adipose tissue p21CIP1+ cells (raw values decreased $17 \%$ by 11 days after completing $D+Q$ treatment). Representative images are shown. (c) $D+Q$ significantly reduced $(p=0.005)$ adipose tissue $S A$ Bgal-expressing cells (raw values decreased by 62\%). Representative images are shown. [Source: EBioMedicine, 2019]. 
This clinical trial suggests that $D+Q$ therapyinduced alleviation of tissue dysfunction and disease progression could potentially benefit complex medical condition such as diabetes and chronic kidney disease (CKD) in humans [8. Administration of $D+Q$ demonstrated significantly reduced $(p=0.001)$ adipose tissue $\mathrm{p} 16^{\mathrm{INK} 4 \mathrm{~A}+}$ cells, where raw values were reduced by $35 \%$ (Figure 2a). Further, senolytics caused a significant reduction $(p=0.009)$ in adipose tissue $\mathrm{p} 21^{\mathrm{ClP} 1+}$ cells as well, with raw values being decreased by 17\% (Figure 2b) [8]. In addition, D + Q also reduced $(p=0.005)$ SABgal $^{+}$cells in which raw values were observed to be reduced by $62 \%$ (Figure 2c) [8]. This human clinical trial shows the senolytics promise of $D+Q$ in decreasing senescent cells that gives the first direct evidence that senolytics are effective in humans [8]. All these preliminary examples of the power of senolytics in anti-ageing therapy show enormous promise to regulate senescence cells and increase healthy lifespans in humans. The first clinical trial of senolytics in human in combating chronic health dysfunctions and age-related diseases gives significant boost to the concept of senolytics based future biomedicine [9].

\section{Conclusion and Outlook}

In general, cellular senescence has been recognized as a hallmark of aging. The consensus is that the deficient clearance of senescent cells by the immune system in aged tissues results in accumulation of senescent cells that subsequently exert deleterious effects and eventually jeopardize tissue homeostasis. The therapeutic perspectives offer a whole host of new opportunities to develop anti-ageing therapies along with therapeutics to battle age-related complex diseases.

The field of senolytics is a relative novel and unexplored research area. Senolytic agents are being investigated for their clinical relevance. We anticipate a rapid growth in the field of senolytics in the near future including clinical trials. It is possible to identify pre-existing clinically-approved antibiotics with senolytic activity, for drug repurposing as anti-aging drugs that can be used to target senescent fibroblasts and other age-related medical conditions. In addition to senolytics, novel methodologies for the detection of cellular senescence and new technologies applied to the analysis of mitochondrial biochemistry are expected to be developed.

\section{References}

1. Baker, D.J., Childs, B.G., Durik, M., Wijers, M.E., Sieben, C.J., Zhong, J., et al., 2016. Naturally occurring p16(Ink4a)positive cells shorten healthy lifespan. Nature $\quad$ 530(7589):184-9. DOI: https://doi.org/10.1038/nature16932

2. Baar M. P., Brandt R. M., Putavet D. A., Klein J. D., Derks K. W., Bourgeois B. R., De Keizer P. L. Targeted apoptosis of senescent cells restores tissue homeostasis in response to chemotoxicity and aging. Cell, 169, 132-147 (2017). DOI:

https://doi.org/10.1016/j.cell.2017.02.031

3. Baker D. J., Wijshake T., Tchkonia T., LeBrasseur N. K., Childs B. G., et. al., Clearance of p16Ink4a-positive senescent cells delays ageing-associated 
disorders. Nature, 479, 232-236 (2011). DOI: https://doi.org/10.1038/nature10600

4. Yi Zhu, Tamara Tchkonia, Tamar Pirtskhalava, Adam C Gower, Husheng Ding, Nino Giorgadze et. al., The Achilles' heel of senescent cells: from transcriptome to senolytic drugs, Aging Cell 14, pp644-658 (2015). DOI: https://doi.org/10.1111/acel.12344

5. Bela Ozsvari, John R. Nuttall, Federica Sotgia, Michael P. Lisanti, Azithromycin and Roxithromycin define a new family of "senolytic" drugs that target senescent human fibroblasts, Aging (Albany NY), (11): 3294-3307 (2018), DOI: 10.18632/aging. 101633

6. [6] Correia-Melo $\mathrm{C}$ et al Rapamycin improves healthspan but not inflammaging in nfkappab1(-/-) mice. Aging Cell 18(1):e12882 (2018), DOI: 10.1111/acel.12882

7. Chen J, Xavier S, Moskowitz-Kassai E, Chen R, Lu CY, Sanduski K, Špes A, Turk $\mathrm{B}$, Goligorsky MS. Cathepsin cleavage of sirtuin 1 in endothelial progenitor cells mediates stress-induced premature senescence. Am J Pathol.; 180:973-83 (2012),

\subsection{6/j.ajpath.2011.11.033}

8. LaTonya J. Hickson, Larissa G.P.Langhi Prata, Shane A. Bobart, Tamara K. Evans, Nino Giorgadze et. al., Senolytics decrease senescent cells in humans: Preliminary report from a clinical trial of Dasatinib plus Quercetin in individuals with diabetic kidney disease, EBioMedicine, 47, 446-456 (2019), DOI: 10.1016/j.ebiom.2019.08.069

9. Jamie N. Justice, N. Justice, Anoop M. Nambiar, Tamar Tchkonia, Nathan K. LeBrasseur, Rodolfo Pascual, Shahrukh K. Hashmi, Larissa Prata, Michal M. Masternak, Stephen B. Kritchevsky, Nicolas Musi, James L. Kirkland, Senolytics in idiopathic pulmonary fibrosis: Results from a first-in-human, open-label, pilot study, EBioMedicine, 40, 554-563 (2019), DOI: 10.1016/j.ebiom.2018.12.052 


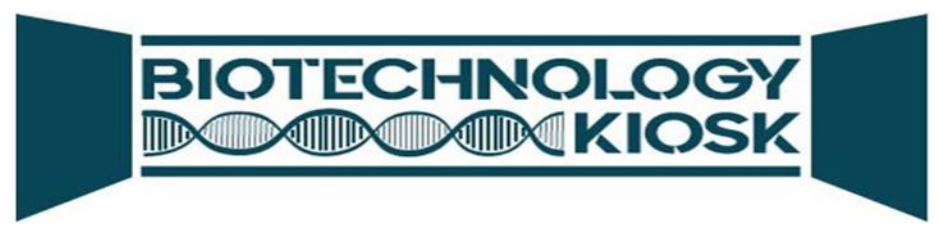

\section{Biotechnology Advances around the World}

\section{Editor's Picks}

Every issue of Biotechnology Kiosk presents select latest research news picked by the editorsin-chief on significant research breakthroughs in different areas of biotechnology around the world. The aim is to promote further R\&D in all of these cutting edge areas of biotechnology. The editors have compiled and included the following innovations and breakthroughs to highlight the recent biotechnology advances.

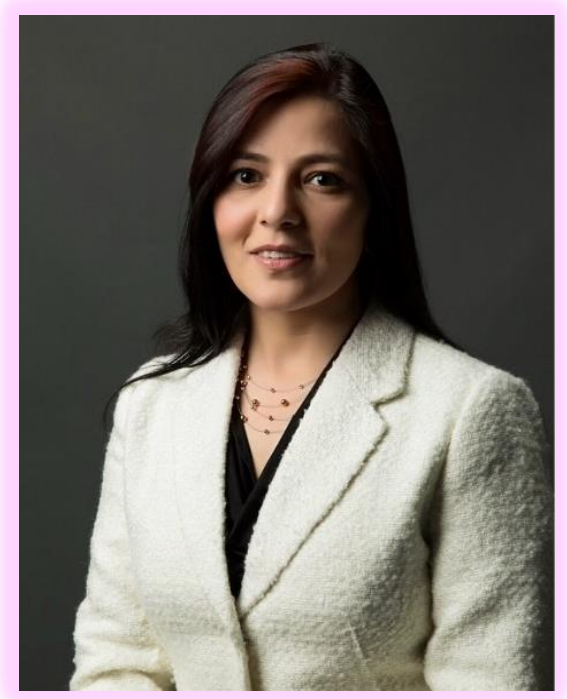

\section{Dr. Megha Agrawal \\ Co Editor-in-Chief}

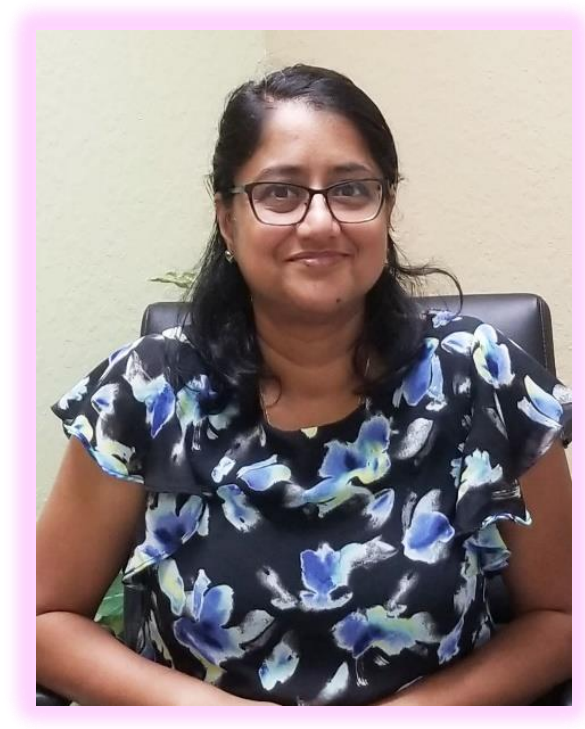

\section{Dr. Shyamasri Biswas \\ Co Editor-in-Chief}




\section{Alzheimer's Disease}

A new diagnosis route based on blood test in the treatment of Alzheimer's disease

One of the major global medical challenges today is the increasing cases of dementia caused by Alzheimer disease (AD). $A D$ is considered a debilitating and incurable disease. It is believed that without the discovery of successful prevention therapies, an estimated 100 million people worldwide will have AD dementia in 2050. In view of this very complex medical challenge, the World Health Organization has initiated a global action plan against dementia that focusses on specifying improved diagnostics as a key area for an optimal disease management and treatment. In this regard, it is believed that an early, accurate, and biomarker-based diagnosis of $A D$ can potentially be very effective along with disease-modifying treatments to prevent and eventually cure AD.

One viable approach for many years has been based on the characterization of amyloid plaques and tau tangles in the brain after a person dies for the effective development of diagnostics to prevent Alzheimer's. Lately, an important aspect that researchers have paid attention to is addressing the discriminative accuracy of plasma phospho-tau217 (P-tau217) for differentiating Alzheimer disease from other neurodegenerative disorders. To this end, development of an inexpensive and widely available blood test for the presence of plaques and tangles is considered a potential breakthrough that would have a profound impact on Alzheimer's research and care.
Recently, a large international team of researchers from the United States, Sweden and Columbia demonstrated a breakthrough approach based on a new blood test that showed remarkable promise in discriminating between persons with and without Alzheimer's disease. This study could pave the way for the diagnosis in persons at known genetic risk and detect the disease as early as 20 years before the onset of cognitive impairment. This research was published in the Journal of the American Medical Association (JAMA) (Discriminative Accuracy of Plasma Phospho-tau217 for Alzheimer Disease vs Other Neurodegenerative Disorders, JAMA, 2020; DOI: 10.1001/jama.2020.12134).

In this new study, researchers measured phospho-tau217 ( $p$-tau217), one of the tau proteins found in tangles, and showed the viable approach of a relatively sensitive and accurate indicator of both plaques and tangles corresponding to the diagnosis of Alzheimer's in living people. Researchers then evaluated a new $p$-tau217 blood test in 1,402 cognitively impaired and unimpaired research participants that comprised from well-known studies in Arizona, Sweden, and Colombia. This study included 81 Arizona participants in Banner Sun Health Research Institute's Brain Donation program who had clinical assessments that provided blood samples in their last years of life and then had neuropathological assessments after they died. This also included 699 participants in the Swedish BioFINDER Study who had clinical, brain imaging, cerebrospinal fluid (CSF), and blood-based biomarker 
assessments. In addition, 522 Colombian autosomal dominant Alzheimer's disease (ADAD)-causing mutation carriers and noncarriers from the world's largest ADAD cohort were also included in the study.

The novel approach of diagnosis based on the $p$-tau217 blood test has great potential in the diagnosis, early detection of Alzheimer's.
Researchers envision that the blood test might become especially useful to improve the recognition, diagnosis, and care of people in the primary care setting that could pave the way to revolutionize Alzheimer's research, treatment and prevention trials, and clinical care.

\section{Anti-Aging Drugs}

\section{Altering activities of protein opens up possibilities for developing anti-aging drugs}

It is known that mitochondria, the energyproducing engine rooms in cells play a crucial role in aging. Mitochondria are essential subcellular organelles for cellular energy production and they also play important functions in a wide array of other cellular processes. These range from cellular signaling to apoptosis. It is believed that functional declines in mitochondria could cause age-related diseases. Previous studies have shown that mild inhibition of mitochondrial respiration could promote longevity in multiple species.

Adenosine 5'-monophosphate (AMP)activated protein kinase (AMPK), which is a critical cellular energy sensor could potentially increase life span in multiple species. AMPK is thought to be one of the factors that is required for the enhanced longevity caused by inhibition of mitochondrial respiration in C. elegans. Hence, it is of huge interest to shed light on the molecular mechanism in $\mathrm{C}$. elegans that may enhance our understanding of human aging and longevity.
Further, the proteins VRK-1 and AMPK have been shown to work in tandem in roundworm cells. In this configuration, VRK1 directs AMPK by sticking a phosphate molecule, which is composed of one phosphorus and four oxygen atoms on it. AMPK monitors energy levels in cells, especially when cellular energy is running low. So, in other words, VRK-1 is thought to regulate AMPK, whereas, AMPK regulates the cellular energy status.

In a recent study, researchers in South Korea were able to dial up and down creatures' lifespans by altering the activity of these proteins found in cells of roundworm $C$. elegans. This process allowed them to convert sugar into energy during low running cellular energy. It has major implications as humans also have these proteins, and thus, this process offers exciting possibilities for developing longevity-promoting drugs. Researchers reported their work in Science Advances (VRK-1 extends life span by activation of AMPK via phosphorylation, Science Advances, 2020; 6 (27): eaaw7824 DOI: 10.1126/sciadv.aaw7824).

In this study, researchers employed a range of different biological research tools, including introducing foreign genes into the 
worm. Subsequently, they were able to dial up and down the activity of the gene to direct the cells to produce the VRK-1 protein. It is interesting to note that throughout the history of evolution, this gene has remained pretty much unchanged, and it is found in most complex organisms including humans. Researchers suggested that the overexpression, or increased production, of the VRK-1 protein boosted the lifespan of the C. elegans. They showed that the inhibition of VRK-1 production reduced its lifespan.

The activity of the VRK-1-to-AMPK cellular-energy monitoring process was found to increase in low cellular energy status by reduced mitochondrial respiration. Researchers attributed the observed results to the set of metabolic chemical reactions that apparently consumed the oxygen from the breathing of worm in order to convert macronutrients from food into the energy.
This energy was available to the cells to spend for their activity.

Interestingly, when similar tinkering was performed with cultured human cells, it was found that those human cells could also replicate this ramping up and down of the VRK-1-to-AMPK process that was observed in roundworms. These significant findings could pave the way for new therapeutic pathway involving VRK-1 to function as a factor in governing human longevity, which could initiate new research and developments of longevity-promoting drugs that alter the activity of VRK-1. This study could also provide new directions for investigating innovative therapeutic strategies to combat metabolic disorders by targeting the modulation of VRK-1. Metabolic disorders involve the disruption of chemical reactions in the body, including diseases of the mitochondria.

\section{Tuberculosis and Antibiotics}

Efficacy of novel compounds against mycobacteria show promise for tuberculosis antibiotics

Tuberculosis (TB) is known to be one of the deadliest infectious diseases, TB is caused by the bacterium Mycobacterium tuberculosis. In recent years, there have been increasing rates of this disease due to rising resistance to the current primary treatment regimen. This includes directly observed treatment, short-course and also decreasing efficacy of the BCG vaccine. The World Health Organization (WHO) global data show a grim picture of the mortality rate from TB that reveal each day over 4000 people die from TB and 300,000 people fall ill from the disease.

Infectious disease researchers around the world have put in significant efforts to develop new treatments for TB. However, for the most part, these treatments have been shown to be unsuccessful with the current first-line treatments that have been in use for over 50 years. Further, on a related front, antimicrobial resistance (AMR) is believed to be now as one of the biggest problems facing us in the in the 21st Century. Without an effective solution to AMR, there could be epidemics of bacterial disease going forward. 
In a recent study, researchers in the U.K. and Switzerland tested novel compounds for their potential as antibiotics and demonstrated promising activity against TB. They evaluated two compounds with antibacterial properties, which had been produced by the company Redx Pharma as antibiotic candidates, particularly against TB. Their research appeared in the Journal of Antimicrobial Chemotherapy (Structural and mechanistic analysis of ATPase inhibitors targeting mycobacterial DNA gyrase, Journal of Antimicrobial Chemotherapy, 2020; DOI: 10.1093/jac/dkaa286).

Their strategy in the search for new treatments was based on to finding novel compounds in order to exploit well-known existing targets for drugs such as the bacterial enzyme DNA gyrase. It is known that the member of the DNA topoisomerase family of enzymes is required for bacterial DNA functionality. This makes these compounds that inhibit its activity much sought after as antibiotic candidates.
The research team then employed Xray crystallography to elucidate the molecular details of the action of the compounds against their target. This revealed a very common mutation in DNA gyrase, which causes bacteria to be resistant to a related group of antibiotics. However, it was found that aminocoumarins did not lead to resistance to the compounds that were under study.

Researchers showed that the Redx03863-binding pocket was distinct from that of novobiocin. This feature coupled with the lack of activity of resistant mutants suggested that such compounds could have potential to be further exploited as antibiotics.

Further, they envisioned that taken together with the relatively small size of Redx03863 and Redx04739 along with their ease of synthesis, and their activity against Gram-negative and Gram-positive bacteria, including $M$. tuberculosis, these novel compounds could be very promising for the treatment of TB.

\section{Stem Cells and Regenerative Therapies}

\section{Chaperone-mediated autophagy in embryonic stem cells provides new therapeutic targets to repair or regenerate damaged cells and organs}

It is known that there are over 200 different types of specialized cells in human bodies. All these specialized cells are possible to derive from embryonic stem (ES) cells. Studies have shown that these ES cells relentlessly self-renew while retaining the ability to differentiate into any cell type in adult animals. This state is known as pluripotency. While, it is known that these cells' metabolism plays a role in this process, researchers have tried to understand the mechanism of working of the cells' internal wiring that helps keep pluripotency and how it ultimately decides stem cell fate. Further, there is a significant interest to gain insights into chaperone-mediated autophagy (CMA) that corresponds to a process of a cell-eating mechanism, which is necessary for survival and function of most living organisms. There are a few forms of autophagy that are known 
to be present in all eukaryotic cells. CMA belong to the autophagy that is unique to mammals and the physiological role of CMA is not yet understood fully.

During the self-eating process of cells, the intracellular materials are delivered to lysosomes that are organelles, which help break down these materials. In a recent study, researchers in the US addressed this self-eating process in embryonic stem cells and a related metabolite to explore the possibility of serving as promising new therapeutic targets to repair or regenerate damaged cells and organs. The new preclinical study that was published in Science (Chaperone-mediated autophagy regulates the pluripotency of embryonic stem cells, Science, 2020, DOI: 10.1126/science.abb4467) showed for the first time how the stem cells keeps CMA at low levels to promote that self-renewal. Also, they provided new insights into how the ready stem cell switches that suppression off to enhance CMA, among other activities, and differentiate into specialized cells.

This study resulted in a significant discovery in the field of stem cell biology that seeks to develop therapies for tissue or organ regeneration. In this study, researchers demonstrated two novel ways based on CMA and a metabolite to potentially manipulate the self-renewal and differentiation of stem cells. The metabolite is known as alphaketoglutarate and shown to be regulated by CMA. They showed that rationally intervening or guiding these functions could provide a new innovative way to increase the efficiency of regenerative medicine approaches.
Researchers employed metabolomic and genetic laboratory techniques on the embryonic stem cells of mice to better understand significant changes that took place during their pluripotent state and subsequent differentiation. In this process, CMA activity was observed to be kept at a minimum due to two cellular factors critical for pluripotency including Oct4 and Sox2. These cellular factors can suppress a gene known as LAMP2A, which is thought to provide instructions for making a protein called lysosomal associated membrane protein-2 necessary in CMA. Researchers showed that the minimal CMA activity allowed stem cells to maintain high levels of metabolite alphaketoglutarate that was crucial to reinforce a cell's pluripotent state.

During the time for differentiation, the cells were shown to begin to upregulate CMA due to the reduction in Oct4 and Sox2. Subsequently, augmented CMA activity led to the degradation of key enzymes that were responsible for the production of alphaketoglutarate. This resulted in a reduction in alpha-ketoglutarate levels as well as an increase in other cellular activities to promote differentiation.

These important research findings reveal that CMA and alpha-ketoglutarate can dictate the fate of embryonic stem cells. The role of autophagy in the stem cell could pave the way to new breakthroughs lead to better therapies to treat various disorders.

\section{Compiled and Edited by Dr. Megha Agrawal \& Dr. Shyamasri Biswas}

Co Editors-in-Chief 


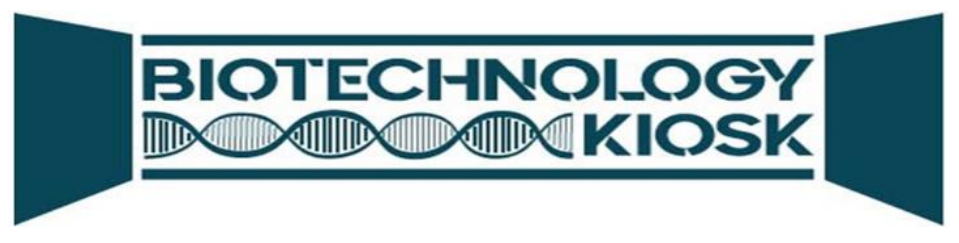

\section{Biotech and Pharma Industry Roundup}

CytoSorbents awarded $\$ 4.4$ Million Grant by the DoD for Universal Plasma Product

CytoSorbents Corporation was recently awarded a $\$ 4,421,487$ threeyear contract by the U.S. Department of Defense (DoD) to complete preclinical development of its HemoDefend-BGA plasma and whole blood adsorber. The advantage of this filter is that it is designed to allow a "universal plasma" that can be given to anyone no matter their blood type. The process essentially involves removing the anti-A and anti-B antibodies from the blood. This basically makes the blood "O" type that can be given to anyone

[Source:

\section{https://www.biospace.com/].}

Sangamo, Novartis join hands to develop genetic medicines for autism, other brain disorders

In a recent announcement, Sangamo Therapeutics and Novartis agreed to work together to develop treatments that aim to alter the expression of key genes implicated neurodevelopmental disorders like autism

[Source: https://www.biopharmadive.com/].

\section{Supply of Gilead's COVID-19 drug remdesivir secured by Europe}

In a recent announcement, Gilead agreed to supply European Union countries with enough doses of the COVID-19 medicine remdesivir to treat about 30,000 patients. In this agreement, European authorities can also secure more doses of the drug, which was the first authorized in the EU to treat the disease caused by the new coronavirus. The Supply of this drug are currently tight, with the U.S. reserving nearly all available doses in July

[Source: https://www.biopharmadive.com/].

\section{Eastman Kodak to help manufacturing drugs, vaccines in the US}

In order to make sure that important medicines are produced in the U.S., the Trump administration is taking bold 
and decisive steps to enlisting two manufacturers to help manufacture drug ingredients as well as experimental coronavirus vaccine candidates. In a recent announcement, the U.S government disclosed plans to loan Eastman Kodak $\$ 765$ million under the Defense Production Act, funding a pivot by the company to manufacture active pharmaceutical ingredients for generic drugs

[Source: https://www.biopharmadive.com/].
\$2.1 billion deal involving GSK and Sanofi with U.S. Government for COVID-19 Vaccine

The U.S. government's Operation Warp Speed program made a deal with GlaxoSmithKline and Sanofi struck with to supply an initial 100 million doses of a COVID-19 vaccine candidate under development by the two companies. In its initial analysis of the deal, Forbes announced that the price of a vaccine comes out to about $\$ 10.50$ per dose [Source: https://www.biospace.com/].

\section{AlloVir Raises \$276 Million in IPO to Advance Cell Therapy Programs}

The US based AlloVir recently raised \$276 million in an upsized initial public offering. The funds will be used to advance the company's efforts to develop an allogeneic cell therapy to control infections

in immunocompromised patients. AlloVir, formerly known as ViraCyte, is known for its expertise in developing allogeneic, off-the-shelf virus-specific $\mathrm{T}$ Cell (VST) therapy candidates targeting 12 devastating viruses, including COVID-19 [Source: https://www.biospace.com/].

\section{COVID-19 Vaccine Candidate into Phase I by Merck in Q3}

Joining the ranks of other pharmaceutical companies, Merck recently announced a COVID-19 vaccine candidate in clinical development. This vaccine candidate will move in the third quarter of this year according to the announcement by Merck. This move to Phase I happened as a result of the acquisition of Austria-based Themis by Merck, which has a pipeline of vaccine candidates and immune-modulatory therapies. Subsequently, Merck started the vaccine development program with its May acquisition of the company, which is developing V591, a 
SARS-CoV-2 vaccine candidate that uses a measles virus vector platform based

on

a

vector

[https://www.biospace.com/].

Roche's IL-6 receptor blocker fails in Phase III trial

In a recent announcement, Roche reported the failure of its IL-6R antagonist tocilizumab in patients with COVID-19. This puts the efficacy of IL6 inhibition at question. In the COVACTA global Phase III trial, it was reported that Roche's approved standard medicine for blocking cytokine release syndrome (CRS) did not meet the primary endpoints of improving the clinical status of COVID19 patients with pneumonia and of reduction in mortality. It is to be noted that

previously, Sanofi's/Regeneneron' s $\quad$ IL-6 signalling blocker sarilumab also failed to reduce mortality and to reduce disease severity in mechanically ventilated COVID-19 patients [Source: https://european-biotechnology.com/].

COVID-19 vaccine being jointly developed by Pfizer and BioNTech's shows positive data

Pfizer (New York based) and BioNTech (Germany based) pharma companies recently announced positive results obtained from the preliminary data from the most advanced of four of its vaccine candidates against SARS-CoV-2. The vaccine program (BNT162) is focusing on at least four vaccines, in which each a different combination of mRNA format and target antigen is employed for each vaccine candidate. The preliminary data, which is announced is for BNT1621b1. This vaccine encodes an optimized SARS-CoV-2 receptor binding domain (RBD) antigen [Source: https://www.biospace.com/].

\section{A New H1N1 Strain from China could possibly trigger another global pandemic}

Researchers have observed that another pandemic could be building in China with a new strain of the H1N1 swine flu that has been reported already jumped to humans. The new strain, G4 EA H1N1 was revealed to emerge on China's pig farms According to the researchers, this strain has all the hallmarks of a pandemic virus, which is a recently emerged genotype 4 (G4) reassortant Eurasian avian-like (EA) $\mathrm{H} 1 \mathrm{~N} 1$ virus that bears 2009 pandemic (pdm/09) and triple-reassortant (TR)-derived internal genes [Source: https://www.biospace.com/].

Menlo Therapeutics' ZILXI ${ }^{\mathrm{TM}}$ (minocycline) topical foam, $1.5 \%$ receives FDA approval for the treatment of inflammatory lesions of rosacea in adults 
Rosacea is known as a diverse skin condition. The most commonly symptoms of rosacea are deep facial redness, spider veins (telangiectasia) and acne-like inflammatory lesions (papules and pustules). Menlo Therapeutics Inc., which is a specialty pharmaceutical company focused on therapies to address unmet needs in dermatology recently announced the approval of ZILXITM (minocycline) topical foam, $1.5 \%$ by the FDA. ZILXI ${ }^{\mathrm{TM}}$ is used for the treatment of inflammatory lesions of rosacea in adults. ZILXI was developed as FMX103 by Menlo's wholly-owned subsidiary Foamix Pharmaceuticals Ltd. ("Foamix"), is considered the first minocycline product of any kind to be approved by the FDA for use in rosacea. This FDA approval is considered very encouraging news for clinicians and patients seeking options for this difficult to treat skin disorder [Source: https://www.biospace.com/].

\section{Meningococcal Vaccines expected to touch US $\$ 9$ billion by 2026}

Bacteria Neisseria meningitides causes meningococcal disease. This disease occurs throughout the world especially in the developing world. Typical symptoms include inflammation in the membranes of the brain and spinal cord which is characteristic of meningitis. The meningococcal vaccines market is pretty vast with a lot of potential to make its footprints even larger in the future that is expected to reach $\$ 9$ billion by 2026 . This vaccine exists in the market and doing a great business due to its utility and need for preventing the incidence of this deadly disease

[Source:

https://www.biospace.com/].

\section{Price set for COVID-19 drug remdesivir}

In a recent announcement, the manufacturer of the drug remdesivir, Gilead said it will charge between $\$ 2,340$ and $\$ 3,120$ for a typical course of treatment with its COVID-19 drug remdesivir. In recent clinical trials, this drug has been shown to speed the recovery of patients hospitalized with the infectious disease. In the plan announced by Gilead, it will offer remdesivir to governments in developed countries at a price of $\$ 390$ per vial. However, in the U.S., private insurers, as well as government health programs like Medicare that don't purchase drugs directly, will pay $\$ 520$ per vial [Source: https://www.biopharmadive.com/].

\section{WHO proposes plan to deliver 2 billion coronavirus vaccine doses}

The World Health Organization, together with partner organizations announced an ambitious plan to secure 2 billion doses of coronavirus vaccines by the end of 2021 . This plan is aimed to ensure high-risk groups around the world that can have access to any vaccine that's successfully developed [Source: https://www.biopharmadive.com/)].

\section{Large scale phase 2 and phase 3 trials are underway for coronavirus vaccine}


Late-stage clinical trials are underway involving tens of thousands of people for coronavirus vaccine development. Phase 2 and phase 3 trials are being prepared in the U.S., Europe and also other regions due to the fact that the pandemic's heaviest impact has begun to shift to the Southern Hemisphere. This large scale clinical trials correspond to an important transition from an early safety studies of coronavirus vaccines that involved several dozens to hundreds of healthy volunteers. But considering the uniqueness of this global pandemic that will require safety and effectiveness of a treatment to be potentially given to tens of millions of people globally, massive trials spanning many countries are required. To this end, a phase 2 study of a vaccine being developed by the University of Oxford and AstraZeneca aims to recruit 10,260 adults and children in the U.K. In addition, the British drug maker is planning a phase 3 trial in the U.S. involving about 30,000 people [Source: https://www.biopharmadive.com/)].

\section{Investors pouring cash to counter cancer genes}

It is known for years that a family of genes that, when mutated, turn regular cells cancerous. There has been recent progress in developing drugs that can effectively silence those genes. This has fueled investor optimism in companies going after these genes that are known as RAS. The most recent example of investments comes from a California based biotech company named Revolution Medicines that has raised \$238 million through an initial public offering. RAS genes are known to make special proteins that regulate how a cell grows, specializes and divides. One of these proteins, K-ras, has drawn a particularly large amount of attention for R\&D. and Amgen, the country's largest biotech has released data showing an experimental drug appeared to stabilize disease in a small group of lung cancer patients with KRAS gene mutations. This has further fueled extensive R\&D in this area [Source: https://www.biopharmadive.com/)].

\section{EC pledges $€ 4.9 \mathrm{bn}$ to grant access to medicines}

Under the Coronavirus Global Response Framework, the European Commission recently announced to allocate further $€ 4.9 \mathrm{bn}$ to assure access to COVID-19 countermeasures for poor countries [Source: https://european-biotechnology.com/].

\section{No beneficial effect of the antiviral HIV treatment lopinavir-ritonavir in patients hospitalized with COVID-19}

Recent clinical data from the UK's RECOVERY trial demonstrated no clinical benefit from use of lopinavir-ritonavir in hospitalised COVID-19 patients [Source: https://european-biotechnology.com/].

\section{Dutch Biotech Company HALIX B.V. to produce vaccines against COVID- 19}

Dutch CDMO HALIX B.V. has entered in a research consortium coordinated by the University of Oxford, to provide GMP- 
compliant production of Vaccitech Ltd's COVID-19 vaccine (ChAdOx1 nCoV-19) targeting the viral spike protein [Source: https://european-biotechnology.com/].

\section{SARS-CoV2 antibody tests might} not be suitable for individual diagnosis

An international research consortium in Europe has provided data that demonstrate that SARS-CoV-2 antibody tests are only useful for population-based analyses and might not work for individual diagnosis. [Source:

https://european-

\section{Enthera Pharmaceuticals Srl secures $€ 28 \mathrm{~m}$ in Series A financing}

In a recent announcement, Italian start-up Enthera Pharmaceuticals reported securing $€ 28 \mathrm{~m}$ to advance restorative therapy for type 1 diabetes and inflammatory bowel disease. The startup announced that it will use the proceeds to advance its pipeline, Ent001, an IGFBP3/TMEM219 blocker, to clinical proof of principle (Phase lb) for type 1 diabetes, gastrointestinal diseases [Source: https://european-biotechnology.com/]. biotechnology.com/]. 


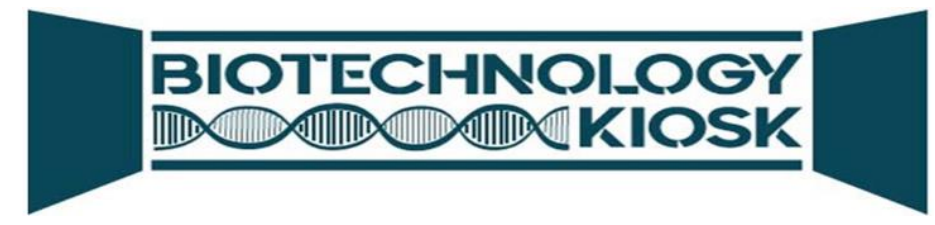

For all production related questions or sending your ads, please email or call our production department: E-mail: sales@biotechkiosk.com; Phone: 386-518-9411.

THANK YOU! 